\title{
On the model coefficients for the standard and the variational multi-scale Smagorinsky model
}

\author{
By JOHAN MEYER $S^{1,2}$ AND PIERRE SAGAUT ${ }^{1}$ \\ ${ }^{1}$ Laboratoire de Modélisation en Mécanique, Université Pierre et Marie Curie - Paris 6, \\ Boîte 162, 4 place Jussieu, 75252 Paris cedex 05, France \\ ${ }^{2}$ Department of Mechanical Engineering, Katholieke Universiteit Leuven, Belgium \\ meyers@lmm.jussieu.fr, johan.meyers@mech.kuleuven.be; sagaut@lmm.jussieu.fr
}

(Received 28 November 2005 and in revised form 8 June 2006)

A theoretical analysis is presented on the behaviour of the model coefficients for the well-known Smagorinsky model and two variational multi-scale (VMS) variants of the Smagorinsky model. The dependency on two important parameters is addressed, i.e. the ratio of the LES-filter width $\Delta$ and the Kolmogorov scale $\eta$ on the one hand, and the ratio of the integral length scale $L$ and the LES-filter width $\Delta$ on the other hand. First of all, it is demonstrated that the model coefficients vary strongly with $\Delta / \eta$. By evaluating the model coefficients as functions of the subgrid activity $s$ (which expresses the relative contribution of the subgrid-scale model in the total dissipation, and corresponds to a nonlinear transformation of $\Delta / \eta$ ), we show that a classical Lilly-Smagorinsky model overestimates the dissipation, even in cases where the dissipation of the subgrid-scale model is dominant. Therefore, generic and easy-to-use modifications to the different models are proposed, which provide close approximations to the models employing 'exact' coefficients. For the standard Smagorinsky model, this modified model corresponds to approximating the eddy viscosity $v_{t}$ as $v_{t}=\left(v_{\text {Lilly }}^{2}+v^{2}\right)^{1 / 2}-v$, with $v_{\text {Lilly }}$ the turbulent viscosity obtained by employing Lilly's classical Smagorinsky constant and $v$ the laminar viscosity. Similar easy-to-use relations are presented for the variational multi-scale Smagorinsky models. Next to the $\Delta / \eta$ dependence of the model coefficients, the $L / \Delta$ behaviour is also elaborated. Although a strong dependence on $L / \Delta$ is observed for low values of the ratio, we do not advocate the use of $L / \Delta$-dependent model coefficients. Rather, the asymptotic $L / \Delta$ independence and the speed of asymptotic convergence are used as a tool to compare the quality of subgrid-scale models (e.g. $L / \Delta>10$ is a minimum order of magnitude for the small-small VMS model), and differences are observed between the standard Smagorinsky model and its two VMS variants. Finally, for the VMS models, the influence of the shape of the high-pass filter, used in the variational multi-scale formulation, is investigated. We observed that smooth high-pass filters result in more robust VMS Smagorinsky models.

\section{Introduction}

Large-eddy simulations (LES) are now firmly established on an academic level as a research and design tool for turbulent flows. There have been several comprehensive reviews (see e.g. Lesieur \& Métais 1996; Moin \& Kim 1997; Ghosal 1999; Pope 2004), and some first text books (e.g. Sagaut 2002; Geurts 2003) on the subject. The continuing work on this subject, slowly pushes LES to a mature technology for the 
simulations of fluid-flow phenomena; but even so, several issues still remain open. One of the more important issues is the closure of the subgrid-scale stresses by means of a suitable model.

Based on the definition of a low-pass filter in space, the LES equations can be derived by filtering the Navier-Stokes equations. The aim of this operation is to reduce the temporal and spatial complexity of high-Reynolds-number turbulence, such that its numerical simulation is feasible on modern day computing facilities. It is well accepted that the direct numerical simulation (DNS) of the Navier-Stokes equations of many relevant turbulent flows remains computationally unmanageable for the next few decades (Jiménez 2003). However, the low-pass filtering of the nonlinear terms in the Navier-Stokes equations introduces a set of closure terms, the subgrid-scale stresses, which cannot be explicitly expressed in terms of filtered variables. In fact, these subgrid-scale stresses account for the interaction between the - by virtue of the filter - unresolved scales and the resolved LES variables. At present, the modelling of these subgrid-scale stresses in terms of resolved properties remains an important problem in large-eddy simulations.

Naturally, the formulation of a suitable subgrid-scale closure should ensure that the behaviour of mean flow properties and flow statistics resulting from large-eddy simulations remain comparable to Navier-Stokes turbulence. Regrettably, as reviewed by Jiménez \& Moser (2000), we can estimate, based on conditional averages, that the statistical error between the 'best' model based on resolved properties and the effective subgrid-scale stresses is at the least in the order of $80 \%$. This clearly illustrates the difficulty related to the formulation of subgrid-scale closures.

In practice, we can devise a ranking of properties a subgrid-scale model should contain or preserve. Certainly, the first item on such a list is the requirement that the model enacts the correct level of total turbulent dissipation. Without suggesting a specific order, items further down the list might contain the preservation of symmetry properties of the differential equations, the inclusion of back-scatter effects, the prediction of intermittency levels, realizability, etc. (see e.g. Meneveau 1994; Vreman, Geurts \& Kuerten 1994; Fureby \& Tabor 1997; Ghosal 1999; Carati, Winckelmans \& Jeanmart 2001, for more information). The quality of a subgrid-scale closure can be roughly appreciated based on the number of physical and mathematical properties it effectively preserves.

According to physics, the problem of the subgrid closure can be considered in a different way. It is well known from experiments that turbulent flows become Reynolds independent at very large Reynolds numbers. This so-called asymptotic Reynolds behaviour of flows is related to the turbulent dissipation occurring at very small scales (order of magnitude of the Kolmogorov scale), while the important flow features are determined by the large energy-containing scales of turbulence. Both regions, are typically separated by a turbulent inertial subrange. At very high Reynolds numbers, the separation between the dissipation scales and the large scales in turbulence is so large, that the dissipation mechanism no longer influences the flow features. In a sense, accurate LES of any asymptotic-Reynolds-number flow can be performed by simulating the corresponding 'lowest' asymptotic-Reynolds-number case with a resolution corresponding to the Kolmogorov scale of the lower Reynolds number. As a result of the scale separation, the selected dissipation mechanism, or - using LES nomenclature - subgrid-scale closure, which is used at the smallest scales will not influence the mean flow solution. For the 'lowest' asymptotic Reynolds number, we can estimate that the effective ratio between the large scales with length $L$ and the Kolmogorov scale $\eta$, i.e. $L / \eta$, amounts at least to 1000 (Jiménez 2003). In fact, 
for many practical applications, asymptotic Reynolds numbers are high and resolving the Kolmogorov scale of the 'lowest' asymptotic Reynolds number is effectively beyond the capability of modern day computers. Hence, in view of these reflections, a good subgrid-scale model for LES should succeed in providing an effective scale separation between the dissipation mechanism (at scales of the order of the LES low-pass filter width $\Delta$ ) and the large turbulent scales (with length $L$ ) for values of $L / \Delta$ which are much lower than the lowest $L / \eta$ ratio of asymptotic turbulence. Naturally, lower values of $L / \Delta$ for which the subgrid-scale model should still provide scale separation, will impose more stringent restrictions on the formulation of the subgrid-scale closure and the inclusion of a number of requirements contained in the above-mentioned ranking of important model properties might be necessary. In fact, in our opinion, the ratio $L / \Delta$ at which the model dissipation mechanism and the large turbulent scales start to be mutually independent can be an interesting objective measure for the quality of a subgrid-scale model. This line of thought will be further explored in the present paper.

The most well-documented subgrid model in LES literature is the Smagorinsky (1963) model. The model was developed with very high-Reynolds-number applications in mind. It formulates a turbulent viscosity, using the assumption that turbulence beyond the LES filter width is in equilibrium, such that the production and dissipation of subgrid energy are balanced. Lilly (1967) presented a theoretical analysis, which determines - for high Reynolds numbers - the model coefficient associated with the Smagorinsky model. Muschinski (1996) took this analysis further and introduced the Smagorinsky fluid, a virtual fluid, containing a Smagorinsky viscosity instead of laminar viscosity. In this study, a relation between the Smagorinsky coefficient and the LES filter shape was introduced. Later, Magnient, Sagaut \& Deville (2001) verified Muschinski's framework with actual numerical simulations of a Smagorinsky fluid. Verifying the general shape of the eddy viscosity for different LES filters, Leslie \& Quarini (1979) showed that the Smagorinsky model indeed formulates an eddy viscosity which is - on average - close to the exact spectral eddy viscosity. Hence, various theoretical studies support the Smagorinsky model as an interesting LES closure. Also from a physical point of view, taking the discussion in the previous paragraph in mind, the Smagorinsky closure should yield accurate results provided that $L / \Delta$ is large enough and provided that the turbulence is indeed in equilibrium at subgrid scales.

Nevertheless, practical use of the Smagorinsky model typically reveals that the model is too dissipative in many practical situations, including low-Reynolds-number shear flows, wall-turbulence, transition, etc. In our view, there are two main reasons for this.

(i) In many practical cases, the ratio $L / \Delta$ is too low for the theoretical framework supporting the Smagorinsky model. Specifically, the equilibrium assumption for the subgrid scales is questionable for low values of $L / \Delta$. Canuto \& Cheng (1997) introduced (for high Reynolds numbers) an interesting generalization of the LillySmagorinsky formulation, which does not use the equilibrium assumption, and naturally recovers specific large-scale behaviour of stratification and shear. Using a different approach, Hughes, Mazzei \& Jansen (2000) proposed a variational multiscale formulation of the Smagorinsky model, which restricts the effect of the subgridscale dissipation to the smallest resolved scales of the simulation. This model has been shown to provide better results than the standard Smagorinsky model in various practical applications (see e.g. Hughes, Mazzei \& Oberai 2001a; Hughes, Oberai \& Mazzei 2001b; Holmen et al. 2004). Naturally, by deviating from the classical 
Smagorinsky formulation, many different subgrid formulations attempt to provide good results for low values of $L / \Delta$.

(ii) The Smagorinsky coefficient is not a constant, contrary to what Lilly's (1967) analysis suggests. As will be shown in the present paper, the Smagorinsky coefficient strongly depends on the local ratio of the filter width $\Delta$ to the Kolmogorov scale $\eta$. Even for moderate to relatively high Reynolds-number flows, this influence can be important (e.g. a priori results in the present paper indicate that the standard LillySmagorinsky model can overestimate dissipation with approximately $5 \%$ to $10 \%$ for a subgrid activity - here defined as the ratio of the subgrid-scale-model dissipation to the total dissipation - in the order of 0.95 and 0.9 , whereas this error can be up to $50 \%$ at lower values for the subgrid activity). Voke (1996), while implicitly assuming $L / \Delta=\infty$, presented a first framework showing that the Smagorinsky coefficient depends on the grid Reynolds number (which is related to $\Delta / \eta$ ). Regrettably, his modifications to the Smagorinsky coefficient are not widely used, possibly because his proposal is based on fits which are not generic and include a parameter which depends on the shape of the energy spectra which were deployed during his analysis. In the same context, Meneveau \& Lund (1997) found for the dynamic procedure that the $\Delta / \eta$ dependence of the Smagorinsky coefficient can be important in simulations. They demonstrated that the application of the classical dynamic procedure (Germano et al. 1991), does not correctly reproduce this dependence as it assumes a coefficient which is the same at the LES-filter and test-filter level.

The present paper introduces a framework which allows us to evaluate the model coefficients of both the standard Smagorinsky model and the variational multi-scale (VMS) formulations of the Smagorinsky model as functions of both the ratio $L / \Delta$ and the ratio $\Delta / \eta$ and this for a general class of low-pass filters (note that, for high Reynolds numbers, the product of $L / \Delta$ with $\Delta / \eta$ corresponds to the Reynolds number, by virtue of the well-known relation $\left.\operatorname{Re} \sim(L / \eta)^{4 / 3}\right)$. Consequently, the model coefficient's dependence on these parameters can be evaluated. It will be shown that both $L / \Delta$ and $\Delta / \eta$ can have a very large influence on the effective model coefficient. However, the dependence on these two parameters will be used in a different way.

First of all, as discussed above, it is the $L / \Delta$ independence of the model coefficient which interests us from a practical point of view. How fast the asymptotic independence of the model's coefficient for $L / \Delta \gg 1$ is reached, will be shown to be an interesting point of reference for comparing the standard Smagorinsky model to the different variational multi-scale Smagorinsky formulations. It is, however, not our intention to proclaim the direct use of $L / \Delta$-dependent model coefficients in simulations. In fact, dependence of the coefficients on $L / \Delta$ for $L / \Delta \sim 1$ corresponds to our particular choice for the low-wavenumber behaviour of turbulence, i.e. homogeneous isotropic turbulence, and different choices for the large-scale behaviour of the turbulence will yield different results. However, we assume that the main trends observed for the asymptotic behaviour and the speed of this asymptotic convergence, which we use as a point of reference for comparing the models, are not too sensitive to the behaviour of the large-scale turbulence.

Secondly, the $\Delta / \eta$ dependence of the model coefficients will be shown to be important, and - in contrast to the $L / \Delta$ behaviour - we will advocate the use of modified models which account for this dependence. Resulting from our theoretical analysis, which expresses the model coefficient's dependence on the relevant flow parameters, a methodology is proposed, which allows the construction of generic and accurate approximations to the 'exact' models (i.e. the models which use a coefficient with the exact dependence on $\Delta / \eta)$. Good approximations are presented for the 
standard Smagorinsky model and for two versions of the variational multi-scale formulation of the Smagorinsky model. As a preview of these results, we will show that such an approximation for the Smagorinsky model corresponds to calculating the eddy viscosity $v_{t}$ in the LES as $v_{t}=\left(v_{\text {Lilly }}^{2}+v^{2}\right)^{1 / 2}-v$, with $v_{\text {Lilly }}$ the turbulent viscosity obtained by employing Lilly's model constant and $v$ the laminar viscosity. One can clearly appreciate the ease of implementation and the generic nature of this proposal. In the course of the present text, the origin of this approximation will be rigorously justified.

The paper is organized in the following way. First, in $\S 2$, the standard Smagorinsky model in analysed, the behaviour of the model's coefficient is evaluated and improved formulations for the Smagorinsky model are elaborated. In $\S 3$, a similar formalism is elaborated for the variational multi-scale formulation of the Smagorinsky model. Furthermore, the VMS and standard Smagorinsky models are compared based on their dependence on $L / \Delta$. Finally, conclusions are presented in $\S 4$.

\section{An analysis of the standard Smagorinsky model}

As introduced in the previous section, large-eddy simulations are formally based on the low-pass filtered Navier-Stokes equations. As a point of reference, we define the (one-dimensional) low-pass filter operation $\mathscr{G}$ as

$$
\bar{f}(y)=\mathscr{G} f=\int K_{\mathscr{G}}\left(y-y^{\prime}\right) f\left(y^{\prime}\right) \mathrm{d} y^{\prime}
$$

where $K_{\mathscr{G}}(y)$ is the filter kernel. Further, $\mathscr{G}$ has the property that $\mathscr{G}_{c}=c$, for every constant function $c$. The Fourier transform of the above filter definition corresponds to $\bar{f}(k)=G(k) f(k)$, with $G(k)$ the transfer function associated with $K_{\mathscr{G}}(y)$.

Filtering the Navier-Stokes equations introduces a closure problem, i.e. the subgridscale stresses $\tau_{i j}$ have to be expressed using a model $m_{i j}$ which is based on filtered flow variables only:

$$
m_{i j} \rightarrow \tau_{i j}=\overline{u_{i} u_{j}}-\bar{u}_{i} \bar{u}_{j}
$$

A general class of models often used in LES are eddy-viscosity models. They model the deviatoric part of $\tau_{i j}$ and correspond to

$$
m_{i j}=-2 v_{t} \bar{S}_{i j}
$$

Here, $v_{t}$ is the turbulent viscosity or eddy viscosity. Further, the filtered rate of strain tensor $\bar{S}_{i j}=\left(\partial \bar{u}_{i} / \partial x_{j}+\partial \bar{u}_{j} / \partial x_{i}\right) / 2$. An often used expression for the eddy viscosity, introduced by Smagorinsky (1963), reads

$$
v_{t}=C_{s}^{2} \Delta^{2}\left(2 \bar{S}_{i j} \bar{S}_{i j}\right)^{1 / 2},
$$

where $C_{s}$ is the Smagorinsky coefficient and $\Delta$ the filter width related to the filter $\mathscr{G}$. Hence, using the short-hand notation $|\bar{S}|=\left(2 \bar{S}_{i j} \bar{S}_{i j}\right)^{1 / 2}$, the Smagorinsky model reads

$$
m_{i j}=-2 C_{s}^{2} \Delta^{2}|\bar{S}| \bar{S}_{i j} .
$$

In the present section, the behaviour of the model coefficient $C_{s}$ of the Smagorinsky model as a function of the ratio $L / \Delta$ and as a function of the Reynolds number is evaluated. Results of this analysis will demonstrate that the theoretical behaviour of $C_{s}$ in many practical simulations is actually far from constant. Hence, our choice of the term Smagorinsky coefficient instead of the more commonly used 'Smagorinsky constant'. 
The remainder of this section is further organized in three parts. In $\S 2.1$, Lilly's (1967) classical expression for the Smagorinsky coefficient is briefly reviewed. In $\S 2.2$, more general relations for the Smagorinsky coefficient as a function of $L / \Delta$ and the Reynolds number are elaborated and evaluated. In $\$ 2.3$, an easy-to-implement approximation is constructed which is shown to be a considerable improvement over the classical 'constant-coefficient' Smagorinsky model.

\subsection{The Lilly value for the Smagorinsky coefficient}

In early literature, $C_{s}$ was often hypothesized to be a universal constant related to the Smagorinsky model. Different values for $C_{s}$ are quoted, ranging from 0.1 (Deardoff 1970), 0.15 (Pope 2000), 0.17 (McMillan \& Ferziger 1979), to 0.17 (Lilly 1967), 0.185 and 0.23 (Lilly 1966). Some of these values are a result of an empirical fit of the Smagorinsky model to DNS results (Deardoff 1970; McMillan \& Ferziger 1979). In contrast, Lilly (1966, 1967), Muschinski (1996) and Pope (2000), for example, determined the Smagorinsky model on theoretical considerations.

The classical determination of a theory-based value of the Smagorinsky model makes two assumptions. First, an infinite Reynolds number is assumed, i.e. molecular viscosity is neglected. Further, one approximates

$$
v_{t} \approx C_{s, \infty}^{2} \Delta^{2}\left\langle 2 \bar{S}_{i j} \bar{S}_{i j}\right\rangle^{1 / 2}
$$

The difference from (2.4) being the ensemble averaging operator $\langle\cdot\rangle$ which is introduced on the norm of the strain-rate tensor. Experience has shown that the introduction of this ensemble averaging operator indeed has no relevant influence on the performance of the model. For instance, McMillan \& Ferziger (1979) were among the first to verify this. Using both these assumptions, we can express the dissipation of energy as

$$
\varepsilon=\left\langle-m_{i j} \bar{S}_{i j}\right\rangle=\left(C_{s, \infty} \Delta\right)^{2}\left(2 \int_{0}^{\infty} k^{2} \bar{E}(k) \mathrm{d} k\right)^{3 / 2},
$$

with $\bar{E}(k)$ the LES-filtered energy spectrum. Lilly (1967) chose the LES filter to be a sharp cutoff filter with cutoff $k_{c}=\pi / \Delta$ and further assumed $E(k)$ to be an inertial range spectrum, i.e. $E(k)=\alpha \varepsilon^{2 / 3} k^{-5 / 3}$. Combining this with (2.7) and some algebra yields

$$
C_{s, \infty}=\frac{1}{\pi}\left(\frac{2}{3 \alpha}\right)^{3 / 4} .
$$

For $\alpha=1.5$, which is generally accepted as the value for the Kolmogorov constant, this leads to $C_{s, \infty}=0.173$. For the remainder of this paper, relation (2.8) will be used as a definition for $C_{s, \infty}$.

\subsection{The Smagorinsky coefficient for finite Reynolds numbers}

As an alternative to an inertial-range energy spectrum, a more sophisticated spectrum can be used for $E(k)$, such as, for example, that proposed by Pope (2000)

$$
E(k)=\alpha \varepsilon^{2 / 3} k^{-5 / 3} f_{L}(k L) f_{\eta}\left(k L R e_{L}^{-3 / 4}\right),
$$

with

$$
\begin{aligned}
& f_{L}(x)=\left(\frac{x}{\left[x^{2}+c_{L}\right]^{1 / 2}}\right)^{11 / 3} \\
& f_{\eta}(x)=\exp \left(-c_{\beta}\left(\left[x^{4}+c_{\eta}^{4}\right]^{1 / 4}-c_{\eta}\right)\right)
\end{aligned}
$$


Further, $L$ is the integral length scale, defined by $E^{3 / 2} / \varepsilon$ (with $E$ the total energy) and $R e_{L}$ is the Reynolds number based on $L$, defined by $R e_{L}=E^{1 / 2} L / v$. Moreover, $c_{\beta}=5.2$, and $c_{L}, c_{\eta}$ are positive parameters which depend on the Reynolds number. In fact, $c_{L}, c_{\eta}$ are determined by ensuring that $\int_{0}^{\infty} E(k) \mathrm{d} k=E$ and $\int_{0}^{\infty} k^{2} E(k) \mathrm{d} k=\mathscr{E}$, with $\mathscr{E}$ the total enstrophy. More details on the determination of $c_{L}, c_{\eta}$ and $c_{\beta}$ can be found in Pope (2000) and Meyers \& Baelmans (2004).

In the present paper, some practical a priori evaluations will be performed using the specific definitions (2.10) and (2.11) for $f_{L}$ and $f_{\eta}$. These spectra are well established and comparisons with a wide range of experimental data were provided (Pope 2000, p. 235). The universal small-scale part of the energy spectrum, generated by $f_{\eta}$, provides accurate fits to experiments in, for example, wake turbulence, homogeneous shear, jets, boundary layers and pipe flows, for Taylor-Reynolds numbers ranging from 30 to 1500 . Obviously, the large-scale part of the spectrum, generated by $f_{L}$, is specific to the large-scale flow type, and is constructed to suit homogeneous isotropic turbulence. Therefore, whenever evaluations in the present study depend on this lowwavenumber region of the spectrum, we will carefully discuss the validity of any related conclusions.

We want to emphasize that the majority of theoretical derivations in the present study do not depend on a specific definition for $f_{L}$ and $f_{\eta}$ such as those provided in (2.10) and (2.11), which are mainly used for actual numerical evaluations. Any suitable energy spectrum of the form (2.9) with appropriate definitions for $f_{L}$ and $f_{\eta}$ can be used, as long as it includes an explicit dependence on the integral length scale $L$ and the Reynolds number, and provided it guarantees the important properties $\int_{0}^{\infty} E(k) \mathrm{d} k=E$ and $\int_{0}^{\infty} k^{2} E(k) \mathrm{d} k=\mathscr{E}$.

Based on an appropriate analytical description of the spectrum which is Reynoldsnumber dependent, a more general relation can be derived for $C_{s}$ at finite Reynolds numbers. To this end, (2.7) is generalized by adding a laminar viscosity part, leading to

$$
\begin{aligned}
\varepsilon & =\varepsilon_{t}+\varepsilon_{v}=\left(C_{s} \Delta\right)^{2}\left(2 \int_{0}^{\infty} k^{2} \bar{E}(k) \mathrm{d} k\right)^{3 / 2}+2 v \int_{0}^{\infty} k^{2} \bar{E}(k) \mathrm{d} k, \\
& =\left(C_{s} \Delta\right)^{2}\left(2 \int_{0}^{\infty} k^{2}(G(k))^{2} E(k) \mathrm{d} k\right)^{3 / 2}+2 v \int_{0}^{\infty} k^{2}(G(k))^{2} E(k) \mathrm{d} k,
\end{aligned}
$$

with $\varepsilon_{t}$ the dissipation generated by the Smagorinsky model and $\varepsilon_{v}$ the dissipation generated by laminar viscosity. Note that in (2.12) the product $(G(k))^{2} E(k)$ is positive, so that the integrals in (2.12) are positive. Further, it is self-evident that $0 \leqslant \varepsilon_{v} \leqslant \varepsilon$, such that $C_{s}^{2} \geqslant 0$ is guaranteed. In (2.12), we further assume that the filter operation $\mathscr{G}$ is spherical symmetric in Fourier space, such that its effect can be incorporated based on a one-dimensional transfer function acting on the sphere-averaged energy spectrum. Some generalizations for filters which are not spherical symmetric in Fourier space (as encountered in various practical implementations) are added in the Appendix.

To proceed, we first introduce the auxiliary function $\Phi$, which will be extensively used, i.e.

$$
\Phi\left(\frac{L}{\Delta}, R e_{L}\right)=\frac{\int_{0}^{\infty} x^{1 / 3}(G(x / L))^{2} f_{L}(x) f_{\eta}\left(x R e_{L}^{-3 / 4}\right) \mathrm{d} x}{\int_{0}^{\infty} x^{1 / 3}(G(x / L))^{2} \mathrm{~d} x},
$$

with the auxiliary variable $x$ corresponding to $k L$. The function $\Phi$ is normalized such that it evolves to 1 if $\Delta \ll L$ and $\Delta \gg \eta$, where $\eta$ is the Kolmogorov scale 
$\left(\eta=\left(v^{3} / \varepsilon\right)^{1 / 4}\right)$. In this case, when compared to the extensive inertial range, where $f_{L}=f_{\eta}=1$, the contributions of low- or high-wavenumber ranges of the spectrum where $f_{L}<1$ or $f_{\eta}<1$, are negligible in the total integration in (2.13). Note that $\Delta / \eta \gg 1$ and $L / \Delta \gg 1$ further implies that the filter $\mathscr{G}$ cuts in the inertial subrange and that $R e_{L} \gg 1$.

In order to represent the normalization of $\Phi$ in a simpler and more useful form, we will introduce a new parameter $\gamma$, i.e.

$$
\gamma=\frac{\left(\frac{4}{3} \int_{0}^{\infty} k^{1 / 3}(G(k))^{2} \mathrm{~d} k\right)^{3 / 4}}{\pi / \Delta},
$$

which depends only on the shape of the filter $\mathscr{G}$. For a sharp cutoff filter with cutoff wavenumber $k_{c}=\pi / \Delta$, we can easily verify that $\gamma=1$. By introducing the definition for $\gamma$ in (2.13), we obtain

$$
\Phi=\frac{4}{3} \frac{\int_{0}^{\infty} x^{1 / 3}(G(x / L))^{2} f_{L}(x) f_{\eta}\left(x e_{L}^{-3 / 4}\right) \mathrm{d} x}{(\gamma \pi L / \Delta)^{4 / 3}} .
$$

Using the auxiliary function (2.15), and the definitions $L=E^{3 / 2} / \varepsilon$ and $R e_{L}=$ $E^{1 / 2} L / v$ into equation (2.12) leads to

$$
1=\gamma^{2} C_{s}^{2}\left(\frac{3 \alpha}{2}\right)^{3 / 2} \pi^{2} \Phi^{3 / 2}+\left(\frac{3 \alpha}{2}\right) \frac{(\gamma \pi L / \Delta)^{4 / 3}}{R e_{L}} \Phi
$$

Whence,

$$
C_{s}=\frac{C_{s, \infty}}{\gamma} \Phi^{-3 / 4} \sqrt{1-\left(\frac{\gamma L}{C_{s, \infty} \Delta}\right)^{4 / 3} \frac{1}{R e_{L}} \Phi .}
$$

By introducing the well-known relation $R e_{L}=(L / \eta)^{4 / 3}$ - the equality (instead of the more generally encountered proportionality) in this relation is ensured by the careful definition of $R e_{L}$ and $L$ - where $\eta$ represents the Kolmogorov length scale, we further obtain

$$
C_{s}=\frac{C_{s, \infty}}{\gamma} \Phi^{-3 / 4} \sqrt{1-\left(\frac{\gamma \eta}{C_{s, \infty} \Delta}\right)^{4 / 3} \Phi} .
$$

In this expression, $C_{s}$ depends on the ratio $L / \Delta$ and on the Reynolds number.

Some obvious limits can now be verified. First of all, for $R e_{L} \rightarrow \infty$ and $L / \Delta$ constant (i.e. also $\Delta / \eta \rightarrow \infty$ ), we obtain

$$
C_{s}\left(\frac{L}{\Delta}, \infty\right)=\frac{C_{s, \infty}}{\gamma} \Phi\left(\frac{L}{\Delta}, \infty\right)^{-3 / 4} .
$$

When in addition $L / \Delta \gg 1$, it follows that $C_{s}=C_{s, \infty} / \gamma$.

Two limits which are not so straightforward, are, for constant Reynolds number $\operatorname{Re}_{L}$, the limit $L / \Delta \rightarrow \infty$ (and hence $\Delta / \eta \rightarrow 0$ ), and the limit $L / \Delta \rightarrow 0$. These limits can be readily expressed for a sharp cutoff filter with cutoff $k_{c}=\pi / \Delta$. To this end, we can simplify $\Phi$ to

$$
\Phi_{s c}=\left(\frac{C_{s, \infty} \Delta}{\gamma L}\right)^{4 / 3} \operatorname{Re}_{L}\left[1-\frac{2 \alpha}{R e_{L}} \int_{\pi L / \Delta}^{\infty} x^{1 / 3}(G(x / L))^{2} f_{L}(x) f_{\eta}\left(x \operatorname{Re}_{L}^{-3 / 4}\right) \mathrm{d} x\right] .
$$


For $L / \Delta \rightarrow \infty$ and $R e_{L}$ constant (and hence also $\Delta / \eta \rightarrow 0$ ), the term in square brackets converges exponentially to 1 , since the energy in the tail of the spectrum decays exponentially for increasing wavenumbers (cf. the formulation of $f_{\eta}$ in (2.11)). Inserting this into (2.17), allows us to obtain $C_{s} \rightarrow 0$ for this limit. For the second limit, $L / \Delta \rightarrow 0$ (and $R e_{L}$ constant), elaboration provides $\Phi_{s c} \rightarrow 0$, such that $C_{s} \rightarrow \infty$. It is, however, important to remark that this second limit is related to our selection of the large-scale flow behaviour, and, for example, for flows with a mean shear, this limit will be different.

For general smooth low-pass filters, the situation is more subtle, and both limits depend on the shape of the filter $\mathscr{G}$. To obtain the first limit $(L / \Delta \rightarrow \infty)$ a Taylor-series expansion of $G(x / L)$ around $\Delta$ can be used, i.e. $G(x / L) \approx 1-C^{\prime}(x \Delta / L)^{q}$, with $q$ the order of the filter and $C^{\prime}$ a constant. Using this in (2.15) and (2.17) allows us to find that $C_{s} \rightarrow 0$ if $q>2$. For $q=2$, the limit is finite, whereas $q<2$ yields $C_{s} \rightarrow \infty$. The second limit is related to the decay of the filter transfer function at high wavenumbers. If we take $G(x / L) \lesssim(x \Delta / L)^{-r}$, then $G(x / L)$ should decay faster than $r=2 / 3$ for the limit to correspond to $C_{s} \rightarrow \infty$.

Obviously, apart from the ratio $L / \Delta$ and the Reynolds number, the shape of the LES low-pass filter $\mathscr{G}$ can have a significant influence on the value of $C_{s}$. This is clear from the asymptotic analysis presented above, and further also by the appearance of the parameter $\gamma$ in the equations. For a Gaussian filter $\left(G(k)=\exp \left(-k^{2} \Delta^{2} / 24\right)\right)$, we can verify that $\gamma \approx 1.02$; while for a top-hat filter $(G(k)=2 \sin (-k \Delta / 2) / k / \Delta)$, $\gamma \approx 1.12$. Finally, we recall that for a standard sharp cutoff filter with $k_{c}=\pi / \Delta, \gamma$ is equal to 1. Often, in Smagorinsky implementations, the effect of the LES filter appears only through its filter width $\Delta$, for which definitions might even differ. In this context, $\gamma$ provides a more formal means to account in the determination of the Smagorinsky coefficient for the shape of the LES filter with width $\Delta$. For the LES low-pass filter $\mathscr{G}$, we will - in the present paper - consider only a sharp cutoff filter, as it is not our intent to present an extensive study of the LES filter shape on the model coefficients. Hence, $\gamma=1$, and the above-discussed limits can be expected. Later, in the context of VMS models (cf. §3), we will evaluate some effects of smooth filters, when VMS high-pass filters are considered.

For the sake of further analysis, we introduce the subgrid activity, defined as $s=\varepsilon_{t} / \varepsilon=1-\varepsilon_{v} / \varepsilon$. Based on (2.12) and (2.16), we see directly that

$$
s=1-\left(\frac{\gamma \eta}{C_{s, \infty} \Delta}\right)^{4 / 3} \Phi\left(\frac{L}{\Delta}, R e_{L}\right) .
$$

The term $s$ does not depend directly on $C_{s}$ or the formulation of the Smagorinsky model. This is logical, since the correct selection of $C_{s}$ ensures that the total dissipation of the cutoff filtered spectrum is independent of the filter width $\Delta$ and the model. In a sense, $s$ is a transformation of the variable $\Delta / \eta$. However, where $\Delta / \eta$ allows us to evaluate the change in $C_{s}$ as a function of the proximity between $\Delta$ and the Kolmogorov scale $\eta$, the subgrid activity $s$ allows us to study the evolution of $C_{s}$ as a function of the relative importance of the subgrid-scale dissipation when compared to the total dissipation level. The subgrid activity is often used for the evaluation of large-eddy simulations (see, e.g. Geurts \& Fröhlich 2002; Meyers, Geurts \& Baelmans 2003), and might be often more easily assessed in actual simulations than $\Delta / \eta$.

Based on the effective (numerical) integration of the spectrum functions introduced in equations (2.10) and (2.11), it is possible to evaluate the behaviour of $C_{s}$ a priori. In figure 1 , the evolution of $C_{s}$ is displayed as a function of $s$ for different values of $L / \Delta$. It can be clearly appreciated that $C_{s}$ varies strongly with the subgrid activity. 


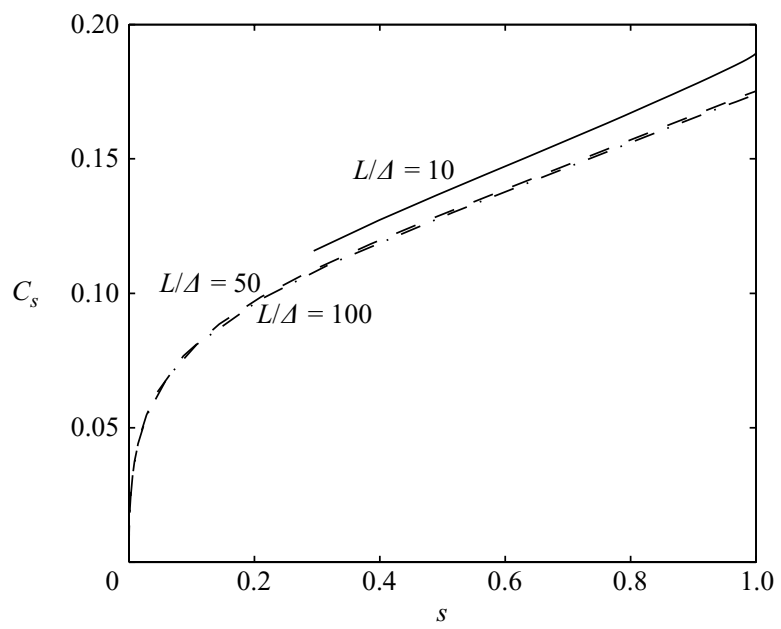

Figure 1. Evolution of $C_{s}$ as a function of the subgrid activity (cf. (2.18) and (2.21)) for different values of $L / \Delta .-, L / \Delta=10 ;---, L / \Delta=50 ;-\cdot-, L / \Delta=100$.

Specifically in the vicinity of $s=1$, which is relevant for many practical LES cases, $C_{s}$ shows a constant slope differing from zero. This illustrates the importance of having a variable 'Smagorinsky constant'. Indeed, in many practical LES of high-Reynoldsnumber applications, one might expect various flow regions where $s$ is considerably lower then 1. Typical examples are jets, mixing layers, wakes, where the local Reynolds number of turbulence is considerably lower outside the mixing regions, or wallbounded flows, where the local turbulent Reynolds number can decrease considerably near the viscous sublayer. Hence, in view of the results presented in figure 1 , it is not surprising that the standard (constant-coefficient) Smagorinsky model is often experienced to be too dissipative.

In figure 1 , the dependence of $C_{s}$ on $L / \Delta$ is also shown. Specifically for $L / \Delta=10$, the Smagorinsky coefficient's dependence on $s$ differs considerably from $C_{s}$ 's behaviour at $L / \Delta=50$ and $L / \Delta=100$. In fact, the latter two values display the $L / \Delta$-asymptotic behaviour of $C_{s}$ when $L / \Delta \gg 1$, while $L / \Delta=10$ is clearly too low. Hence, the Smagorinsky model requires relatively high ratios of $L / \Delta$, before scale separation occurs. This is unsurprising, as it is commonly accepted that the Smagorinsky model often affects the large-scale structures too much. This experience-based observation might be related to the fact that in many practical large-eddy simulations - for lack of computational resources - the ratio of the integral length scales to the LES filter cutoff is often relatively low compared to what would be required for scale separation in a Smagorinsky LES. We will further address the issue of scale separation in $\S 3.3$, where the Smagorinsky model will be compared with some variational multi-scale formulations of the Smagorinsky model.

\subsection{On the formulation of approximations to the exact Smagorinsky coefficient}

In principle, equation (2.18) provides an accurate formula which can be used to determine the Smagorinsky constant. However, in practice, this relation is difficult in use, since properties such as $\Phi$ and $R e_{L}$, or $\Delta / \eta$ are not straightforwardly available during most effective large-eddy simulations. Therefore, a modification of the Smagorinsky model is formulated, which is easy to implement in any NavierStokes solver and which approximates the relation for $C_{s}$ to a certain degree. To achieve this, we will presume $L / \Delta \gg 1$, since, for reasons already discussed above, it is 
not our intention to formulate $L / \Delta$-dependent approximations to (2.18) for practical use. We further recall that the filter $\mathscr{G}$ is a sharp cutoff filter in the present study.

First of all, an approximation to (2.18) which is valid for an LES filter $\mathscr{G}$ which cuts in the inertial subrange, can be obtained by approximating $\Phi$ by its inertial-range value, i.e. $\Phi=1$. In this case,

$$
C_{s}^{\times}=\frac{C_{s, \infty}}{\gamma} \sqrt{\max \left\{1-\left(\frac{\gamma \eta}{C_{s, \infty} \Delta}\right)^{4 / 3}, 0\right\}}
$$

where the max-function is introduced to keep the term under the square-root strictly positive, in case the filter width $\Delta$ is situated in the dissipation range. (The ' $x$ ', and ' $*$ ' superscripts will be used throughout this paper to identify proposed approximations to the 'exact' models.) Obviously, this approximation $C_{s}^{\times}$to $C_{s}$ is not directly usable in simulations, since $\Delta / \eta$ is usually not known. However, if we express the associated turbulent viscosity $v_{t}^{\times}$, we obtain

$$
\begin{aligned}
v_{t}^{\times} & =\left(C_{s}^{\times} \Delta\right)^{2}\left\langle 2 \bar{S}_{i j} \bar{S}_{i j}\right\rangle^{1 / 2}=\max \left\{\left(C_{s, \infty} \Delta / \gamma\right)^{2}\left\langle 2 \bar{S}_{i j} \bar{S}_{i j}\right\rangle^{1 / 2}-v, 0\right\} \\
& =\max \left\{v_{\text {Lilly }}-v, 0\right\} .
\end{aligned}
$$

Clearly, this relation provides an expression which is easy to implement in a NavierStokes solver. It suggests that the turbulent viscosity $v_{t}$ should scale in the inertial subrange and for finite Reynolds numbers as $v_{\text {Lilly }}-v$; this is clearly in contrast to Lilly's Smagorinsky model, which obviously presumes $v_{t}=v_{\text {Lilly }}$ for an inertial-range cutoff of the spectrum.

Evidently, the assumption $\Phi=1$ to obtain (2.22) and (2.23) will not yield a good approximation to $C_{s}$ (cf. (2.18)) when $\Delta$ is approaching the Kolmogorov scale, as in this range $\Phi \neq 1$. Therefore, inspired by (2.23), we will try to formulate a fit to $v_{t}$, which is based on an appropriate combination of $v_{\text {Lilly }}$ and $v$, and which retains the correct scaling of $v_{t}$ in the inertial range. We found this approach successful, as opposed to directly trying to find a fit for $\Phi$ in (2.18), since such a fit of $\Phi$ in practice seems inevitably to require the use of parameters such as the Kolmogorov scale $\eta$.

To this end, define the dimensionless ratios

$$
R=v_{t} / \nu,
$$

and

$$
Q=\left(C_{s, \infty} \Delta / \gamma\right)^{2}\left\langle 2 \bar{S}_{i j} \bar{S}_{i j}\right\rangle^{1 / 2} / \nu
$$

It is now the aim to approximate $R$ based on the property $Q$, i.e. $\mathscr{F}(R, Q)=0$, such that the turbulent viscosity (which corresponds to $R v$ ) approximates well the eddy viscosity of an 'exact' Smagorinsky model (i.e. using the coefficient $C_{s}$ in (2.18)). Moreover, $Q$ is a quantity which is easily obtained in simulations, such that any model which is obtained from a relation $\mathscr{F}(R, Q)=0$ is practical in use. For the Lilly-Smagorinsky model (2.6), we readily see that $R=Q$, while equation (2.23) corresponds to $R=\max \{Q-1,0\}$.

Using (2.18) and the definition of $\Phi$ (2.15), $R$ and $Q$ are expressed as

$$
R=\frac{\left(C_{s} \Delta\right)^{2}\left\langle 2 \bar{S}_{i j} \bar{S}_{i j}\right\rangle^{1 / 2}}{\nu}=\left(\frac{C_{s, \infty} \Delta}{\gamma \eta}\right)^{4 / 3} \Phi\left(\frac{L}{\Delta}, R e_{L}\right)^{-1}-1
$$

and

$$
Q=\frac{\left(C_{s, \infty} \Delta / \gamma\right)^{2}\left\langle 2 \bar{S}_{i j} \bar{S}_{i j}\right\rangle^{1 / 2}}{\nu}=\left(\frac{C_{s, \infty} \Delta}{\gamma \eta}\right)^{4 / 3} \Phi\left(\frac{L}{\Delta}, R e_{L}\right)^{1 / 2}
$$




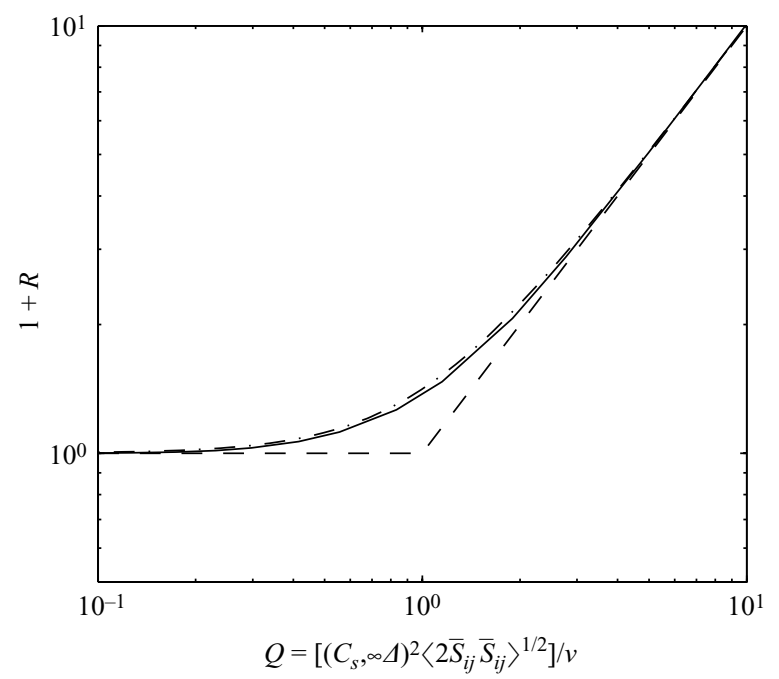

FIGURE 2. Evolution of $R(+1$ to allow logarithmic scaling) as a function of $Q$ for the Smagorinsky model $(L / \Delta \gg 1)$. - $R$ exact; -- - , simple approximation $R^{\times}$of $(2.23) ;-\cdot-$, closer approximation $R^{*}$ corresponding to (2.29).

Hence, based on the integration of $\Phi$, (2.15), employing Pope's spectrum, it is straightforward to evaluate $R$ a priori as function of $Q$. In figure 2 , this theoretical dependence of $R$ as function of $Q$ is presented. Moreover, the relation $R=\max \{Q-1,0\}$, corresponding to $(2.23)$ is also presented.

Based on this analysis, a function $\mathscr{F}$ fitting $R$ to $Q$ can be constructed. We propose to take

which corresponds to

$$
\mathscr{F}(R, Q)=(R+1-Q)(R+1+Q)-1=0,
$$

$$
v_{t}^{*}=\sqrt{\left(C_{s, \infty} \Delta / \gamma\right)^{4}\left\langle 2 \bar{S}_{i j} \bar{S}_{i j}\right\rangle+v^{2}}-v .
$$

In figure 2, this fit is also displayed, and we can appreciate its apparent quality.

In figure 3, the quality of (2.29) is further evaluated. In this figure, the Smagorinsky coefficient $C_{s}^{*}$ associated with (2.29), i.e. $C_{s}^{*}=\left(v_{t}^{*} /\left(\Delta^{2}|\bar{S}|\right)\right)^{1 / 2}$, is evaluated as a function of $\Delta / \eta$ and compared to $C_{s}$ obtained from (2.18). As explained before, we take $L / \Delta \gg 1$, such that results do not depend on $L / \Delta$. (To this end, $L / \Delta=200$ is selected which provides adequate $L / \Delta$ asymptotic behaviour. Consequently, a variation of $\Delta / \eta$ in figure 3 implies a variation of the Reynolds number. One can verify that the range presented in figure 3 (i.e. $1 \leqslant \Delta / \eta \leqslant 100$ ) corresponds to Taylor-Reynolds numbers ranging from about 80 to 1900, which roughly fits in with the range of Reynolds numbers where Pope's spectrum is well established (cf. $\S 2.2$ ).) As a point of comparison, Lilly's Smagorinsky constant, and $C_{s}^{\times},(2.22)$, which is valid in the inertial subrange, are also displayed. In this figure, the quality of $C_{s}^{*}$ as an approximation to $C_{s}$ can be appreciated. We can see that $C_{s}^{*}$ provides a very good approximation to $C_{s}$ for $\Delta / \eta \geqslant 10$. For $4 \leqslant \Delta / \eta \leqslant 10$, the fit deteriorates, but still seems to provide acceptable results. For $\Delta / \eta<4, C_{s}^{*}$ is clearly evolving more slowly to 0 than $C_{s}$. Obviously, this deviation of $C_{s}^{*}$ from $C_{s}$ in this last range is occurring at low values of the model coefficient, such that its importance in the total dissipation balance will be negligible. We will further establish this next, based on an analysis of the induced errors on the turbulent dissipation. 


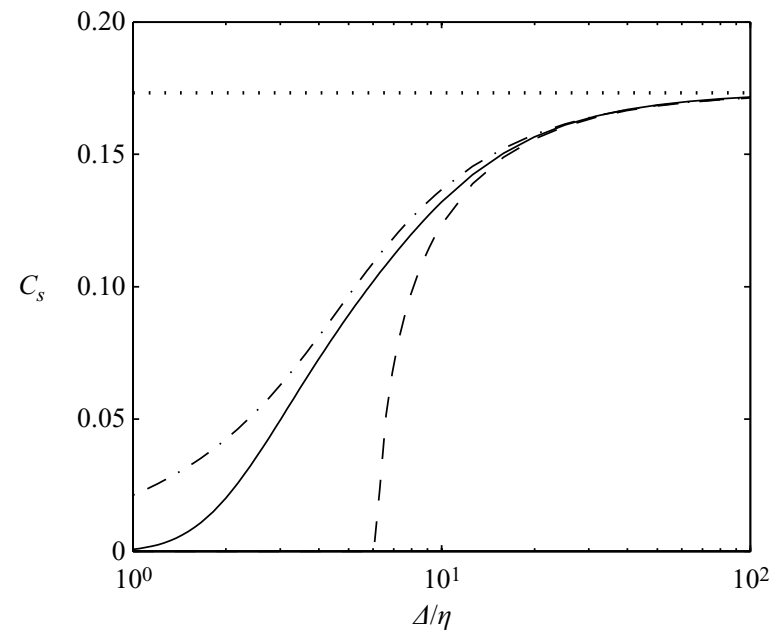

Figure 3. Evolution of $C_{s}$ (2.18), $C_{s}^{\times}$(2.22) and $C_{s}^{*}$ (induced from (2.29)) as a function of the ratio $\Delta / \eta(L / \Delta \gg 1$ is assumed $) .-, C_{s} ;---, C_{s}^{\times} ;-\cdot-, C_{s}^{*} ; \cdots$, Lilly's Smagorinsky constant.

Hence, define

$$
\delta_{\varepsilon}^{a p p r o x}=\frac{\varepsilon_{t}^{\text {approx }}-\varepsilon_{t}}{\varepsilon},
$$

the relative dissipation error generated by an approximation to the 'exact' Smagorinsky model. The subgrid dissipation $\varepsilon_{t}$ of the 'exact' model, i.e. employing $C_{s}$ (cf. (2.18)) is used as a reference in this error definition, and the error is normalized with the total dissipation $\varepsilon$ associated with the 'exact' model. We can readily see that $\varepsilon_{t} / \varepsilon$ corresponds to $s$ (cf. (2.21)). Further, for the standard Lilly-Smagorinsky model, $\varepsilon_{t}^{\text {approx }} / \varepsilon$ can be elaborated as

$$
\frac{\varepsilon_{t}^{\text {Lilly }}}{\varepsilon}=\frac{\left(C_{s, \infty} \Delta / \gamma\right)^{2}\left\langle 2 \bar{S}_{i j} \bar{S}_{i j}\right\rangle^{3 / 2}}{\varepsilon}=\Phi\left(\frac{L}{\Delta}, R e_{L}\right)^{3 / 2} .
$$

For the proposed improvement (cf. (2.29)), we obtain

$$
\frac{\varepsilon_{t}^{*}}{\varepsilon}=\frac{v_{t}^{*}\left\langle 2 \bar{S}_{i j} \bar{S}_{i j}\right\rangle^{1 / 2}}{\varepsilon}=\left[\sqrt{1+\left(\frac{C_{s, \infty} \Delta}{\gamma \eta}\right)^{8 / 3} \Phi\left(\frac{L}{\Delta}, R e_{L}\right)}-1\right]\left(\frac{\gamma \eta}{C_{s, \infty} \Delta}\right)^{4 / 3} \Phi\left(\frac{L}{\Delta}, R e_{L}\right) .
$$

Consequently, $\delta_{\varepsilon}^{\text {Lilly }}$ and $\delta_{\varepsilon}^{*}$ can be evaluated a priori, and results are presented in figure 4, as a function of the subgrid activity. Based on these results, we can clearly quantify the excess in dissipation which can result from a standard Lilly-Smagorinsky model. Even for $s>0.5$, i.e. for regions where the subgrid-scale model provides the dominant part of the dissipation, a considerable overestimation of the dissipation can be observed. At lower values for $s$, this error is even larger, almost reaching $50 \%$. In contrast, the modified model (2.29) has low relative dissipation errors over the full $s$-range with $\delta_{\varepsilon}^{*}<5 \%$.

In the vicinity of $s=1$ and for decreasing $s$ (figure 4), we see that $\delta_{\varepsilon}^{\text {Lilly }}$ increases with a constant slope differing from 0 . This is a direct result of the turbulent viscosity of the Lilly-Smagorinsky model not scaling as $v_{\text {Lilly }}-v$, but obviously as $v_{\text {Lilly }}$. Clearly, 


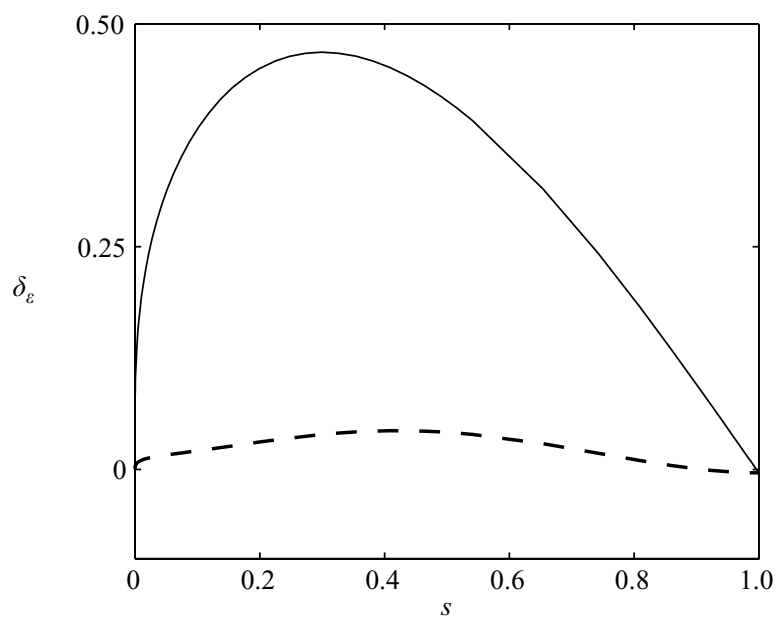

FIGURE 4. Comparison of $\delta_{\varepsilon}^{\text {Lilly }}$ and $\delta_{\varepsilon}^{*}$ as a function of the subgrid activity $s$. ,$- \delta_{\varepsilon}^{\text {Lilly }} ;---, \delta_{\varepsilon}^{*}$.

$\delta_{\varepsilon}^{*}$ has slope zero at $s=1$, and we further verified that this is also the case for the model using $C_{s}^{\times}(2.23)$ (though this last model shows a considerable underprediction of the total dissipation at values $s<0.7$ ).

Obviously, the results in figure 4 are obtained based on an a priori analysis and do not necessarily fully reflect errors on the dissipation in actual simulations. Typically, in cases where the subgrid dissipation is too high, the energy in the tail of the energy spectrum decreases, such that the total dissipation rate, related to the resolved smallscale vorticity, also decreases. However, though errors on total dissipation might appear smaller in actual simulations, the adjustment of the tail of the spectrum will introduce errors in the resolved small-scale LES predictions, while large-scale predictions can also be affected. For instance, in the context of dynamic procedures that do not allow $C_{s}$ to vary between the LES filter and the test filter, Meneveau \& Lund (1997) found errors of $30 \%$ on the magnitude of the subgrid-scale stresses and $10 \%$ on the magnitude of the total stresses at $s \approx 0.3$.

\section{The variational multi-scale Smagorinsky models}

Hughes et al. (2000), in the context of a multi-scale analysis of subgrid-scale modelling for LES, presented the hypothesis that the Smagorinsky model applied to a small-scale extraction of the turbulent field, delivers superior results when compared to a standard Smagorinsky model. In later studies, this was firmly established by effective numerical simulations, see Hughes et al. (2001a) for homogeneous isotropic turbulence, see Hughes et al. (2001b) and Holmen et al. (2004) for a channel flow (see also Jeanmeart \& Winckelmans 2002; Vreman 2003; Stolz, Schlatter \& Kleiser 2005). These observations make the variational multi-scale formulation of the Smagorinsky model interesting subject for further study and comparison with the standard Smagorinsky model.

The formalism presented in $\S 2$, used to analyse and modify the standard Smagorinsky model, can be further extended for variational multi-scale Smagorinsky models. In this section, we will show that the coefficients for VMS Smagorinsky models can be determined based on the same type of a priori analysis. Furthermore, 
modified formulations can be derived which approximate the exact behaviour of the model coefficient. Finally, comparisons between the standard and VMS Smagorinsky model will be presented, which further reveal why and how the VMS formulations are superior to the standard Smagorinsky model.

In their various articles, Hughes et al. present three different versions of the VMS Smagorinsky model, i.e. the large-small, the all-small, and the small-small formulations. Several modifications have been proposed (see, e.g. Jeanmeart \& Winckelmans 2002; Vreman 2003, 2004).

The original formulation of the small-small model corresponds to

$$
m_{i j}=-\left[2 C_{s 1}^{2} \Delta^{2}\left|\bar{S}^{\prime}\right| \bar{S}_{i j}^{\prime}\right]^{\prime},
$$

where the operator $[\cdot]^{\prime}$ is a high-pass filter $\mathscr{H}^{\prime}$. We will not only consider $\mathscr{H}^{\prime}$ to be a high-pass projection operator as proposed by Hughes et al. (2000), but also consider $\mathscr{H}^{\prime}$ to be a more general high-pass filter as proposed by Vreman $(2003,2004)$. Further, $C_{s 1}$ is the model coefficient, which will be shown to depend on $R e_{L}, L / \Delta$, and the shape of the filter $\mathscr{H}^{\prime}$.

Vreman (2003) proposed to discard the outer filter [-]' in (3.1), i.e.

$$
m_{i j}=-2 C_{s 2}^{2} \Delta^{2}\left|\bar{S}^{\prime}\right| \bar{S}_{i j}^{\prime}
$$

Though this proposal is formally not consistent with the original multi-scale framework proposed by Hughes et al. (2000), this model did produce qualitatively comparable results to (3.1) (Vreman 2003), and requires fewer filter operations. If the subgrid dissipation associated with both formulations (3.1) and (3.2) is expressed, we obtain

$$
\begin{aligned}
& \varepsilon_{t, s 1}=2 C_{s 1}^{2} \Delta^{2}\left\langle\left[\left|\bar{S}^{\prime}\right| \bar{S}_{i j}^{\prime}\right]^{\prime} \bar{S}_{i j}\right\rangle=2 C_{s 1}^{2} \Delta^{2}\left\langle\left|\bar{S}^{\prime}\right| \bar{S}_{i j}^{\prime} \bar{S}_{i j}^{\prime}\right\rangle, \\
& \varepsilon_{t, s 2}=2 C_{s 2}^{2} \Delta^{2}\left\langle\left|\bar{S}^{\prime}\right| \bar{S}_{i j}^{\prime} \bar{S}_{i j}\right\rangle .
\end{aligned}
$$

The second equality in (3.3) is valid provided that $\langle\cdot\rangle$ is an appropriate inner product, and that $\mathscr{H}^{\prime}$ is self-adjoint, such that for two variables $f$ and $g,\left\langle f^{\prime} g\right\rangle=\left\langle f g^{\prime}\right\rangle$ (Vreman 2004). In the current study, where the ensemble averaging operator $\langle\cdot\rangle$ is defined as an integration over homogeneous directions in space, and where $\mathscr{H}^{\prime}$ is assumed to be a symmetric convolution filter (i.e. $\left.K_{\mathscr{H}^{\prime}}(y)=K_{\mathscr{H}^{\prime}}(-y)\right)$, this can be easily verified.

Looking at (3.3) and (3.4), the differences between formulations (3.1) and (3.2) seem not very large, and correspond to the presence or absence of a small-scale extraction of the last strain tensor in (3.3).

Next to the small-small model, the all-small formulation (Holmen et al. 2004) of the VMS Smagorinsky model will also be considered in the present study, and it will be interesting to compare this model with the small-small versions. The strict VMS formulation of this model corresponds to

$$
m_{i j}=-\left[2 C_{a 1}^{2} \Delta^{2}|\bar{S}| \bar{S}_{i j}^{\prime}\right]^{\prime} .
$$

The difference with (3.1) is obviously the small-scale extraction which in (3.5) does not appear in the formulation of the magnitude of the strain $|\bar{S}|$, hence the naming all-small, since the strain is obtained using all scales. Again, one might want to discard the outer high-pass filter operation on this model, leading to

$$
m_{i j}=-2 C_{a 2}^{2} \Delta^{2}|\bar{S}| \bar{S}_{i j}^{\prime} .
$$


As for the small-small models, with respect to their induced dissipation, differences between (3.5) and (3.6) are small, and relations similar to (3.3) and (3.4) can be inferred.

The large-small formulation (Hughes et al. 2000) will not be evaluated in the present study, as results and discussions based on the small-small and all-small models will sufficiently reveal relevant trends and adequately demonstrate the methodology.

Further elaboration of the equations will be presented in $\S 3.1$ for the small-small model, and in $\S 3.2$ for the all-small model, resulting in expressions for $C_{s 1}, C_{s 2}, C_{a 1}$ and $C_{a 2}$. Using these relations and a numerical evaluation based on prescribed spectra, a comparison between the standard and VMS Smagorinsky models will be presented in $\S 3.3$ based on their $L / \Delta$ asymptotic behaviour. Subsequently, the $\Delta / \eta$ behaviour of the VMS models will be further investigated in $\S 3.4$. In $\S 3.5$, modifications to the VMS models are proposed and evaluated, which better approximate the 'exact' VMS models, and finally, the effect of the high-pass filter shape on the behaviour of the VMS models is addressed in $\S 3.6$.

\subsection{The small-small formulation}

In the present section, first the original formulation of the small-small model (3.1) is addressed. Afterwards, the formulation (3.2) is briefly surveyed.

In order to further elaborate relations for the model coefficient $C_{s 1}$, an approximation similar to (2.6) has to be introduced. This allows to express the associated subgrid dissipation as

$$
\varepsilon_{t, s 1}=\left(C_{s 1} \Delta\right)^{2}\left\langle 2 \bar{S}_{i j}^{\prime} \bar{S}_{i j}^{\prime}\right\rangle^{3 / 2}=\left(C_{s 1} \Delta\right)^{2}\left(2 \int_{0}^{\infty} k^{2}\left(H^{\prime}(k)\right)^{2} \bar{E}(k) \mathrm{d} k\right)^{3 / 2},
$$

with $H^{\prime}(k)$ the transfer function of the high-pass filter $\mathscr{H}^{\prime}$.

Now a new auxiliary function is defined, similar to the introduction of $\Phi$ in $\S 2.2$, i.e.

$$
\Psi_{1}\left(\frac{L}{\Delta}, R e_{L}\right)=\frac{4}{3} \frac{\int_{0}^{\infty} x^{1 / 3}\left(H^{\prime}(x / L)\right)^{2}(G(x / L))^{2} f_{L}(x) f_{\eta}\left(x R e_{L}^{-3 / 4}\right) \mathrm{d} x}{\int_{0}^{\infty} x^{1 / 3}\left(H^{\prime}(x / L)\right)^{2}(G(x / L))^{2} \mathrm{~d} x} .
$$

This function $\Psi_{1}$ is normalized in such a way that $\Psi_{1}=1$ if $L \gg \Delta \gg \eta$ (i.e. the LES filter $\mathscr{G}$ cuts in the inertial subrange).

Before proceeding with the derivation of an expression for $C_{s 1}$, we will first elaborate a more useful expression for the normalization factor in the denominator of (3.8). In case $\mathscr{H}^{\prime}$ is a high-pass projection filter with (low-wavenumber) cutoff defined as $k_{c}^{\prime}=\pi / \Delta^{\prime}$, where $\Delta^{\prime}>\Delta$, and if the LES filter $\mathscr{G}$ is a sharp cutoff filter with cutoff wavenumber $k_{c}=\pi / \Delta$, then the denominator of (3.8) reduces to

$$
\int_{\pi L / \Delta^{\prime}}^{\pi L / \Delta} x^{1 / 3} \mathrm{~d} x=\frac{3}{4}\left(\frac{\pi L}{\Delta}\right)^{4 / 3}\left(1-\frac{\Delta^{4 / 3}}{\Delta^{1 / 3}}\right)=\frac{3}{4}\left(\frac{\pi L}{\Delta}\right)^{4 / 3}\left(1-\beta^{4 / 3}\right),
$$

where $\beta$ is the LES-filter width to high-pass-filter width ratio $\left(\beta=\Delta / \Delta^{\prime}\right)$, with $0<\beta<1$. In order to use the same expression for a more general class of filters $\mathscr{H}^{\prime}$ and $\mathscr{G}$, we will introduce a correction factor $\gamma_{1}$, which solely depends on the filter shape of $\mathscr{H}^{\prime}$ and the LES filter $\mathscr{G}$, and which is constructed similarly to the definition 
of $\gamma$ (cf. (2.14)), i.e.

$$
\gamma_{1}=\left(\frac{4}{3} \frac{\int_{0}^{\infty} k^{1 / 3}\left(H^{\prime}(k)\right)^{2}(G(k))^{2} \mathrm{~d} k}{(\pi / \Delta)^{4 / 3}\left(1-\beta^{4 / 3}\right)}\right)^{3 / 4}
$$

From the presented definition for $\gamma_{1}$, we readily see that $\gamma_{1}=1$, if $\mathscr{G}$ and $\mathscr{H}^{\prime}$ are respectively a low-pass and a high-pass sharp cutoff filter.

Using (3.7), the auxiliary function $\Psi_{1}$ and the definition of its normalization factors, the small-small version of relation (2.16) can now be constructed, which results in

$$
1=\gamma_{1}^{2} C_{s 1}^{2}\left(\frac{3 \alpha}{2}\right)^{3 / 2} \pi^{2}\left(1-\beta^{4 / 3}\right)^{3 / 2} \Psi_{1}^{3 / 2}+\left(\frac{3 \alpha}{2}\right) \frac{(\gamma \pi L / \Delta)^{4 / 3}}{R e_{L}} \Phi
$$

Consequently, the expression for $C_{s 1}$ reads

$$
\begin{aligned}
C_{s 1} & =\frac{C_{s, \infty}}{\gamma_{1}} \frac{\Psi_{1}^{-3 / 4}}{\left(1-\beta^{4 / 3}\right)^{3 / 4}} \sqrt{1-\left(\frac{\gamma L}{C_{s, \infty} \Delta}\right)^{4 / 3} \frac{1}{R e_{L}} \Phi} \\
& =\frac{C_{s, \infty}}{\gamma_{1}} \frac{\Psi_{1}^{-3 / 4}}{\left(1-\beta^{4 / 3}\right)^{3 / 4}} \sqrt{1-\left(\frac{\gamma \eta}{C_{s, \infty} \Delta}\right)^{4 / 3} \Phi}
\end{aligned}
$$

The $R e_{L}=\infty$ and $L / \Delta \gg 1$ asymptotic behaviour of $C_{s 1}$ can be recovered, yielding

$$
C_{s 1}\left(\frac{L}{\Delta} \gg 1, \infty\right)=\frac{C_{s, \infty}}{\gamma_{1}\left(1-\beta^{4 / 3}\right)^{3 / 4}} .
$$

If we take $\gamma_{1}=1$, corresponding to sharp cutoff filters for $\mathscr{H}^{\prime}$ and $\mathscr{G}$, equation (3.13) corresponds to the relation for $C_{s 1}$ presented by Hughes et al. $(2000,2001 a)$, though using a slightly different nomenclature.

Now, a similar track can be followed to obtain relations for $C_{s 2}$, (3.2), which corresponds to Vreman's proposal to remove the outer high-pass operation in (3.1). To this end, we write

$$
\begin{aligned}
\varepsilon_{t, s 2} & =\left(C_{s 2} \Delta\right)^{2}\left\langle 2 \bar{S}_{i j}^{\prime} \bar{S}_{i j}^{\prime}\right\rangle^{1 / 2}\left\langle 2 \bar{S}_{i j}^{\prime} \bar{S}_{i j}\right\rangle \\
& =\left(C_{s 2} \Delta\right)^{2}\left(2 \int_{0}^{\infty} k^{2}\left(H^{\prime}(k)\right)^{2} \bar{E}(k) \mathrm{d} k\right)^{1 / 2}\left(2 \int_{0}^{\infty} k^{2} H^{\prime}(k) \bar{E}(k) \mathrm{d} k\right) .
\end{aligned}
$$

Consequently, a second auxiliary function has to be defined, corresponding to

$$
\Psi_{2}\left(\frac{L}{\Delta}, R e_{L}\right)=\frac{4}{3} \frac{\int_{0}^{\infty} x^{1 / 3} H^{\prime}(x / L)(G(x / L))^{2} f_{L}(x) f_{\eta}\left(x R e_{L}^{-3 / 4}\right) \mathrm{d} x}{\left(\gamma_{2} \pi L / \Delta\right)^{4 / 3}\left(1-\beta^{4 / 3}\right)}
$$

where

$$
\gamma_{2}=\left(\frac{4}{3} \frac{\int_{0}^{\infty} k^{1 / 3} H^{\prime}(k)(G(k))^{2} \mathrm{~d} k}{(\pi / \Delta)^{4 / 3}\left(1-\beta^{4 / 3}\right)}\right)^{3 / 4}
$$


Further elaboration leads to

$$
\begin{aligned}
C_{s 2} & =C_{s, \infty} \frac{\Psi_{1}^{-1 / 4} \Psi_{2}^{-1 / 2}}{\gamma_{1}^{1 / 3} \gamma_{2}^{2 / 3}\left(1-\beta^{4 / 3}\right)^{3 / 4}} \sqrt{1-\left(\frac{\gamma L}{C_{s, \infty} \Delta}\right)^{4 / 3} \frac{1}{R e_{L}} \Phi} \\
& =C_{s, \infty} \frac{\Psi_{1}^{-1 / 4} \Psi_{2}^{-1 / 2}}{\gamma_{1}^{1 / 3} \gamma_{2}^{2 / 3}\left(1-\beta^{4 / 3}\right)^{3 / 4}} \sqrt{1-\left(\frac{\gamma \eta}{C_{s, \infty} \Delta}\right)^{4 / 3} \Phi} .
\end{aligned}
$$

Obviously, (3.12) and (3.17) do not differ considerably, and both formulations are identical when $\mathscr{H}^{\prime}$ is a projection filter. This is a logical result of the dissipation-based analysis used in the present study to determine the coefficients $C_{s 1}$ and $C_{s 2}$. Naturally, differences in dispersive effects between both formulations are not accounted for, but, as mentioned previously, Vreman (2003) did not discern important differences between simulation results employing these models.

\subsection{The all-small formulation}

Elaborations of the coefficients associated with the all-small models (3.5) and (3.6) are completely equivalent to the procedure followed in the previous section, and no further auxiliary functions have to be introduced. Consequently, both coefficients $C_{a 1}$ and $C_{a 2}$ are readily elaborated to be

$$
\begin{aligned}
C_{a 1} & =C_{s, \infty} \frac{\Phi^{-1 / 4} \Psi_{1}^{-1 / 2}}{\gamma^{1 / 3} \gamma_{1}^{2 / 3} \sqrt{1-\beta^{4 / 3}}} \sqrt{1-\left(\frac{\gamma L}{C_{s, \infty} \Delta}\right)^{4 / 3} \frac{1}{R e_{L}} \Phi} \\
& =C_{s, \infty} \frac{\Phi^{-1 / 4} \Psi_{1}^{-1 / 2}}{\gamma^{1 / 3} \gamma_{1}^{2 / 3} \sqrt{1-\beta^{4 / 3}}} \sqrt{1-\left(\frac{\gamma \eta}{C_{s, \infty} \Delta}\right)^{4 / 3} \Phi} .
\end{aligned}
$$

and

$$
\begin{aligned}
C_{a 2} & =C_{s, \infty} \frac{\Phi^{-1 / 4} \Psi_{2}^{-1 / 2}}{\gamma^{1 / 3} \gamma_{2}^{2 / 3} \sqrt{1-\beta^{4 / 3}}} \sqrt{1-\left(\frac{\gamma L}{C_{s, \infty} \Delta}\right)^{4 / 3} \frac{1}{R e_{L}} \Phi} \\
& =C_{s, \infty} \frac{\Phi^{-1 / 4} \Psi_{2}^{-1 / 2}}{\gamma^{1 / 3} \gamma_{2}^{2 / 3} \sqrt{1-\beta^{4 / 3}}} \sqrt{1-\left(\frac{\gamma \eta}{C_{s, \infty} \Delta}\right)^{4 / 3} \Phi}
\end{aligned}
$$

Clearly, based on the present dissipation analysis of the models, the all-small formulations, (3.18) and (3.19) also do not differ considerably. It can be further observed that the relations for the coefficients of the all-small models (3.18), (3.19) can be obtained by replacing $\Psi_{1}^{-1 / 4}$ in (3.12), (3.17) with $\Phi^{-1 / 4}$ and by adapting the scaling coefficients. However, in the next section, it will be shown that this difference has important consequences for the asymptotic $L / \Delta$ scaling of the respective models.

\subsection{Comparison of VMS and standard Smagorinsky model}

In $\S 1$, the issue of subgrid-scale modelling was discussed in view of the desired scale separation between the large turbulent scales and the dissipation scales. We further suggested that the ratio $L / \Delta$ at which the model dissipation mechanism and the large turbulent scales start to be mutually independent can be a measure to evaluate model quality. In the present section, this idea will be used to investigate and compare the standard, all-small and small-small Smagorinsky models. For this analysis, the filters $\mathscr{G}$ and $\mathscr{H}^{\prime}$ are, respectively, low- and high-pass sharp cutoff filters. Some consequences related to the use of smooth filters will be addressed in $\S 3.6$. 


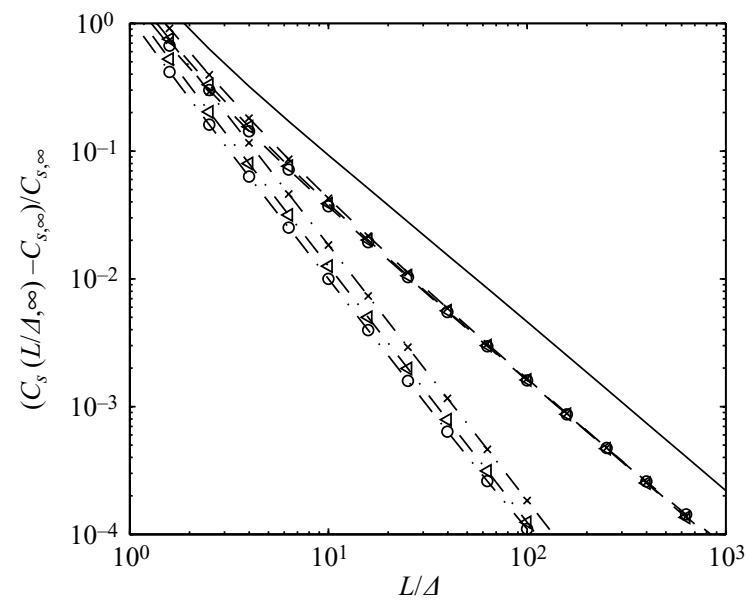

Figure 5. Deviation of $C_{s}, C_{s 1}\left(\times\left(1-\beta^{4 / 3}\right)^{3 / 4}\right)$ and $C_{a 1}\left(\times\left(1-\beta^{4 / 3}\right)^{1 / 2}\right)$ from the asymptotic value $C_{s, \infty}$ as a function of $L / \Delta$ for $R e_{L}=\infty$. The deviations are normalized using $C_{s, \infty}$. ,$- C_{s} ;-\cdot-, C_{s 1} ;---, C_{a 1}$. Symbols further correspond to $\times, \Delta / \Delta^{\prime}=1 / 2 ; \triangleleft, \Delta / \Delta^{\prime}=3 / 4$; $\mathrm{O}, \Delta / \Delta^{\prime}=0.95$.

Before turning to an analysis of those specific models, one might wonder what is a physically relevant minimum for $L / \Delta$. First of all, we can show that scales which are separated by more than a decade do not significantly interact (Kraichnan 1976; Domaradzki \& Rogallo 1990). By further analysing the energy-containing range of Pope's spectrum, we can determine that the turbulent kinetic energy is approximately centred around $0.7 \mathrm{~L}$, while the transition between the energy-containing range and the inertial subrange might be roughly situated at $0.07 \mathrm{~L}$ (Pope 2000). Therefore, as a point of reference in some further discussions, we will use $L / \Delta=10$, such that $L$ and $\Delta$ are separated by a decade and such that $\mathscr{G}$ cuts near the beginning of the inertial subrange.

In order to evaluate the scale separation properties associated with the different models, the behaviour of the different model coefficients as a function of $L / \Delta$ will be evaluated for $R e_{L}=\infty$. In figure 5, the normalized deviation $\left(\left(C_{s}-C_{s, \infty}\right) / C_{s, \infty}\right)$ is displayed as a function of $L / \Delta$ for the coefficients $C_{s}, C_{s 1}$ and $C_{a 1}$. In order, to remove the scaling of both $C_{s 1}$ and $C_{a 1}$ with $\beta$ (cf. equations (3.12) and (3.18)), these coefficients are represented in a normalized way in the figure, by multiplying them, respectively, with $\left(1-\beta^{4 / 3}\right)^{3 / 4}$ and $\left(1-\beta^{4 / 3}\right)^{1 / 2}$.

The results in figure 5 clearly allow us to appreciate the large differences between the three models. First of all, by looking at the level of the deviation around $L / \Delta=10$, deviations are, respectively, $10 \%, 4 \%$ and $1 \%$ for the Smagorinsky model, the best all-small case and the best small-small case. Inversely, by looking at the required ratio for a deviation below an arbitrary selected level of $1 \%$, we obtain, respectively, $L / \Delta \approx 55,25$ and 10, for these three models. Obviously, these evaluations are valid only for homogeneous isotropic turbulence. Nevertheless, we think differences between the models clearly illustrate the higher potential of the VMS Smagorinsky models, when compared to the Smagorinsky model. Further, in this evaluation, the small-small formulation turns out better than the all-small model.

Also interesting in figure 5 are the slopes of the asymptotic convergence associated with the different models. We can see that the convergence for both the Smagorinsky and the all-small model behaves as $(L / \Delta)^{-4 / 3}$, while for the small-small model, the convergence corresponds to $(L / \Delta)^{-2}$. This is a remarkable result, which seems to 
unequivocally single out the small-small formulation as the better model, but calls for a careful analysis of these slopes in view of the large-scale behaviour of the prescribed turbulence implied by Pope's spectrum (cf. (2.10)).

In fact, the $L / \Delta$ asymptotic behaviour of the model coefficients at infinite Reynolds numbers is governed by the dependence of the functions $\Phi$ and $\Psi_{1}$ on $L / \Delta$ (cf. equations (2.17), (3.12), (3.18)). Since the functions $\Phi$ and $\Psi_{1}$ are 1 for $L / \Delta \gg 1$ (and $R e_{L}=\infty$ ), it is particulary interesting to look at their deviation from 1 as a function of $L / \Delta$. We will first concentrate on $\Phi$. At $R e_{L}=\infty$ and a sharp cutoff filter $\mathscr{G}$, the deviation of $\Phi$ from 1 corresponds to

$$
\delta \Phi=\frac{4}{3} \frac{\int_{0}^{\pi L / \Delta} x^{1 / 3}\left(1-f_{L}(x)\right) \mathrm{d} x}{(\pi L / \Delta)^{4 / 3}} .
$$

If we look at the form of the function $f_{L}(x)$, we can see for values $x \gg 1$, that $1-f_{L}(x) \sim x^{-2}$. By generalizing this to $x^{-p}$ (afterwards, we will use $p=2$ for an evaluation of the result) and by taking an arbitrary constant scale $\ell \ll L$ (such that $\left.x_{\ell}=\pi L / \ell \gg 1\right), \delta \Phi$ can be expressed as

$$
\begin{aligned}
\delta \Phi & \approx \frac{4}{3} \frac{\int_{0}^{\pi L / \ell} x^{1 / 3}\left(1-f_{L}(x)\right) \mathrm{d} x+\int_{\pi L / \ell}^{\pi L / \Delta} C x^{1 / 3} x^{-p} \mathrm{~d} x}{(\pi L / \Delta)^{4 / 3}} \\
& =C^{\prime}(L / \Delta)^{-4 / 3}+C^{\prime \prime}(L / \Delta)^{-p},
\end{aligned}
$$

with $C, C^{\prime}$ and $C^{\prime \prime}$ constants which do not depend on $L / \Delta$. Similar derivations can be elaborated for $\delta \Psi_{1}$. By assuming that $\mathscr{H}^{\prime}$ is a high-pass cutoff filter with $\Delta / \Delta^{\prime}$ constant, but $\Delta^{\prime}<\ell$, we readily obtain $\delta \Psi_{1} \sim(L / \Delta)^{-p}$. These relations for $\Phi$ and $\Psi_{1}$ are now further used to obtain the asymptotic behaviour for the different model coefficients given by equations (2.17), (3.12) and (3.18). Some further series expansion yields

$$
\begin{aligned}
\delta C_{s} & \sim(L / \Delta)^{-\min (4 / 3, p)}, \\
\delta C_{a 1} & \sim(L / \Delta)^{-\min (4 / 3, p)}, \\
\delta C_{s 1} & \sim(L / \Delta)^{-p} .
\end{aligned}
$$

Hence, for $p=2$, we recover the slopes observed in figure 5. Moreover, we can appreciate that the slopes of the standard Smagorinsky model and the all-small model are always the same, corresponding to $-4 / 3$ if $p>4 / 3$ and $-p$ otherwise. In contrast, the small-small model always recovers the $-p$ slope.

Naturally, in view of the analysis presented just now, the query for physically relevant values of $p$ arises. Furthermore, we might wonder whether an algebraic rate of decay $p$, as assumed in Pope's spectrum, instead of an exponential decay is justified. First of all, the construction of $f_{L}(2.10)$ is inspired on von Kármán's interpolation formula, which maps a $k^{4}$ low-wavenumber behaviour of the spectrum to the $-5 / 3$ region (in Pope's formulation, a commonly accepted variant, i.e. a $k^{2}$ low-wavenumber dependency is assumed). This interpolation formula is constructed based on the physically expected shape of spectra in the low to moderate wavenumber range. In fact, we can demonstrate that the often experimentally observed exponential decay of the longitudinal velocity correlation functions at high Reynolds numbers, corresponds well with von Kármán's formula for the related spectra (see, e.g. Hinze 1959, pp. 174, 197). Suk Kang, Chester \& Meneveau (2003) considered a generalization of von Kármán's interpolation function, corresponding to $\left(x /\left[x^{p}+c_{L}\right]^{1 / p}\right)^{11 / 3}$. Based 
on their grid-turbulence experiments, they obtained a fit corresponding to $p \approx 1.2$. These observations let us believe that the decay rate of the large-scale influence in the inertial subrange is indeed algebraic, with the order $p$ around 1 or 2 . Note that the above-discussed differences in asymptotic slope between the small-small and both other models, disappear for $p=1.2$ corresponding to Suk Kang et al.'s (2003) fit.

We now turn back to the discussion on the asymptotic behaviour displayed in figure 5, specifically addressing the dependency of the VMS results as a function of $\Delta / \Delta^{\prime}$. Results are shown for $\Delta / \Delta^{\prime}=0.5,0.75$ and 0.95 . Before discussing these results, we want to emphasize that the last value $(0.95)$ is selected in order to investigate the trends for $\beta \rightarrow 1$ predicted by our methodology. However, we do not envisage such an extreme setting of $\beta$ in a practical implementation. Furthermore, we should realize that the $a$ priori methodology used in this paper, is not particulary suited to predicting the real behaviour for such an extreme setting of $\beta$. In fact, the load of a very small part of the turbulence at the tail of the spectrum with the full subgrid dissipation, will certainly distort the shape of the effective spectrum in an unpredictable nonlinear way.

Based on the results in figure 5, we can appreciate that both formulations show the same tendencies with respect to $\Delta / \Delta^{\prime}$. If we look at the small-small model, it is seen that the $(L / \Delta)$ behaviour shifts down with increasing $\Delta / \Delta^{\prime}$. This seems to indicate that $\Delta / \Delta^{\prime} \rightarrow 1$ would produce the best model. However, this will hardly be the case in practice, as $\Delta / \Delta^{\prime} \rightarrow 1$ corresponds to an ever smaller region at the tail of the spectrum on which the model acts. Furthermore from (3.12) and (3.18), we can appreciate that in this case $C_{s 1} \rightarrow \infty\left(C_{a 1} \rightarrow \infty\right)$. In fact, further analysis of the coefficients $C_{s 1}$ and $C_{a 1}$ as a function of $\Delta / \Delta^{\prime}$ in the following subsections, will reveal that values for $\Delta / \Delta^{\prime}$ emerge, beyond which the coefficients display clearly undesirable properties.

In the above analysis, the potential influence of the large-scale turbulence on the model coefficient was evaluated. However, in large-eddy simulations, the scale separation between the large scales and the dissipation mechanism is mainly desired to ensure that the large-scale turbulence in the simulation is not erroneously influenced by the subgrid-scale model. In principle, inversely, the model is allowed to depend on the large-scale solution. Hence, in this sense, the above analysis is too restrictive with respect to the evaluation of scale separation. Nevertheless, the analysis in figure 5 is very relevant in the sense that it isolates the asymptotic $L / \Delta$-region in which the model coefficients can be determined based on the physical behaviour of small-scale turbulence. If one intends to use either the standard Smagorinsky model or the VMS formulations at lower values for $L / \Delta$, it is - in our opinion - not possible to devise a simple algebraic relation for the behaviour of the model coefficient. In such a case, one might consider the approach of Canuto \& Cheng (1997) and incorporate important physical effects of the large-scale flow features directly into the formulation of the model. An alternative solution might employ a double test filter dynamic procedure as proposed by Porté-Agel, Meneveau \& Parlange (2000). They showed that the introduction of an extra test-filter level in the dynamic procedure can be used to account for $L / \Delta$ changes of the Smagorinksy coefficient, and obtained good results in atmospheric boundary-layer flows. Later, a Lagrangian formulation of this procedure was presented by Bou-Zeid, Meneveau \& Parlange (2005).

\subsection{Coefficients of VMS models using a sharp cutoff high-pass filter $\mathscr{H}^{\prime}$}

In this section, we turn towards a further evaluation of the coefficients associated with the VMS models, but now the $\Delta / \eta$ dependence is evaluated, using asymptotic $L / \Delta$ conditions. Further, $\mathscr{H}^{\prime}$ and $\mathscr{G}$ are assumed to be sharp cutoff filters, such that $\gamma=\gamma_{1}=1$. 


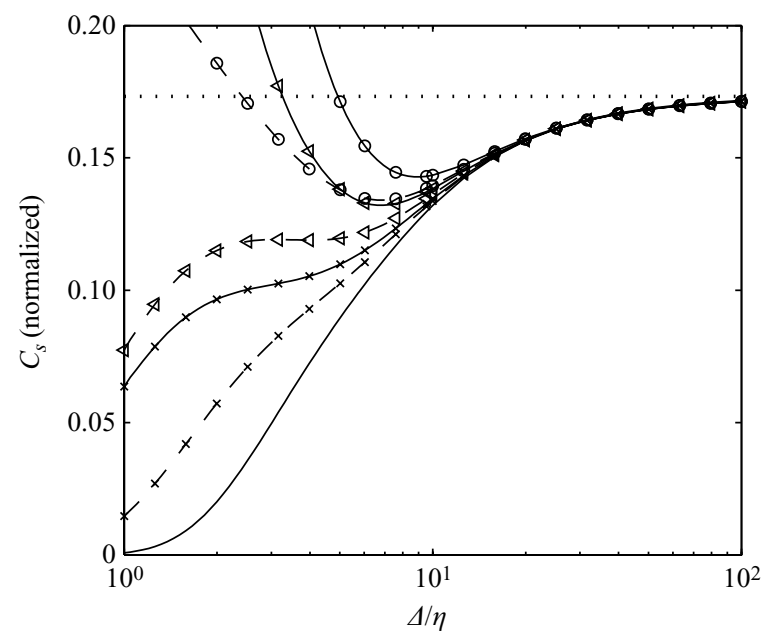

FIGURE 6. Model coefficients $C_{s}, C_{s 1}$ (normalized) and $C_{a 1}$ (normalized) as function of $\Delta / \eta$ (and $L / \Delta \gg 1)$. Without symbols,$- C_{s}(2.18) ; \cdots, C_{s, \infty}$. With symbols -, $C_{s 1}$ (3.12); ,$--- C_{a 1}$ (3.18). Symbols as in figure 5 .

In figure 6, the coefficients $C_{s 1}$ and $C_{a 1}$ (cf. (3.12) and (3.18)) are displayed as a function of the ratio $\Delta / \eta$, for ratios $\Delta / \Delta^{\prime}=0.5,0.75$ and 0.95 . To this end, the functions $\Phi$ and $\Psi_{1}$ were integrated using $L / \Delta=200$, which can be verified (cf. figure 5) to provide $L / \Delta$ asymptotic results. Both coefficients are presented in a normalized way, respectively multiplied with $\left(1-\beta^{4 / 3}\right)^{3 / 4}$ and $\left(1-\beta^{4 / 3}\right)^{1 / 2}$, such that their scaling with $\beta$ does not show in the figure. As a point of reference, the Smagorinsky coefficient $C_{s}$ is also displayed in figure 6, since $C_{s}$ provides the limit $\beta \rightarrow 0$ of $C_{s 1}$ and $C_{a 1}$ (cf. e.g. (2.18) and (3.12)).

For $\Delta / \eta>10$, figure 6 shows that all normalized coefficients, irrespective of $\beta$, collapse onto one curve. For $\Delta / \eta<10$, large differences appear. First of all, we will discuss this range for the all-small coefficient $C_{a 1}$. As seen in the figure, $C_{a 1}$ increases monotonously with $\Delta / \eta$ if $\Delta / \Delta^{\prime}<3 / 4$. When $\Delta / \Delta^{\prime}>3 / 4$ this is not the case any more, and the (normalized) coefficients increase with decreasing $\Delta / \eta$. The latter behaviour occurs, when the high-pass filtered scales used by the model move into the exponential decaying part of the spectrum (at high wavenumbers), while the dissipation which should be provided by the subgrid-scale model is decaying at a slower rate.

The small-small coefficient $C_{s 1}$ shows, in the range $\Delta / \eta<10$, trends which are similar to those discussed for $C_{a 1}$. However, for $C_{s 1}$ the inflection between monotonously decreasing coefficients and increasing coefficients (when $\Delta / \eta$ decreases), can be determined (not shown) to be at $\Delta / \Delta^{\prime} \approx 0.53$. Moreover, at larger values of $\Delta / \Delta^{\prime}$ the increase of $C_{s 1}$ is clearly more pronounced than observed for the all-small coefficient $C_{a 1}$.

\subsection{On the construction of modified VMS models}

In $\S 2.3$, we analysed the inertial range behaviour of $C_{s}$ and the associated turbulent viscosity $v_{t}$ and found that $v_{t} \sim v_{\text {Lilly }}-v(\mathrm{cf}$. (2.22) and (2.23)). By further formalizing this, introducing the ratios $R$ and $Q$, an approximation $\mathscr{F}(R, Q)=0$ to the 'exact' Smagorinsky model was proposed (cf. (2.29)). For the variational multi-scale formulations of the Smagorinksy model, a similar approach can be followed, and we will demonstrate this for $C_{s 2}$ (3.17). 
When the LES filter $\mathscr{G}$ cuts in the inertial subrange, one can replace $\phi, \psi_{1}$ and $\psi_{2}$ in (3.17) by 1 . Consequently, we have in the inertial subrange:

$$
C_{s 2} \sim \frac{C_{s, \infty}}{\gamma_{1}^{1 / 3} \gamma_{2}^{2 / 3}\left(1-\beta^{4 / 3}\right)^{3 / 4}} \sqrt{1-\left(\frac{\gamma \eta}{C_{s, \infty} \Delta}\right)^{4 / 3}} .
$$

We can express a generalized viscosity associated with this model coefficient, and find

$$
\begin{aligned}
v_{t, s 2}^{\prime} & =\left(C_{s 2} \Delta\right)^{2}\left\langle 2 \bar{S}_{i j}^{\prime} \bar{S}_{i j}^{\prime}\right\rangle^{1 / 2} \\
& \sim \frac{\left(\gamma / \gamma_{2}\right)^{4 / 3}}{1-\beta^{4 / 3}} \times\left[\left(\frac{C_{s, \infty} \Delta}{\gamma}\right)^{2}\left(\frac{\left(\gamma / \gamma_{1}\right)^{4 / 3}\left\langle 2 \bar{S}_{i j}^{\prime} \bar{S}_{i j}^{\prime}\right\rangle}{1-\beta^{4 / 3}}\right)^{1 / 2}-v\right] .
\end{aligned}
$$

Here, $v_{t, s 2}^{\prime}$ is the generalized viscosity, where the prime in the notation is added to distinguish it from a classical viscosity which is multiplied with a normal strain tensor instead of with a high-pass filtered strain tensor. In (3.26), various scaling factors appear, which allow us to correctly balance the dissipation effects of parts in the expression which have a different dimensionality. First of all, we can see that $\Delta^{2}\left\langle\bar{S}_{i j}^{\prime} \bar{S}_{i j}^{\prime}\right\rangle^{1 / 2}$ is normalized with a factor such that it can correctly be combined with the viscosity $v$. Further, the full term in square brackets is multiplied by a second factor, such that the total result effectively has the dimensionality of $v_{t, s 2}^{\prime}$.

Identifying these factors in (3.26), allows us to straightforwardly define

$$
R_{s 2}=\frac{v_{t, s 2}^{\prime}}{v} \frac{1-\beta^{4 / 3}}{\left(\gamma / \gamma_{2}\right)^{4 / 3}},
$$

and

$$
Q_{s 2}=\frac{\left(C_{s, \infty} \Delta / \gamma\right)^{2}}{v}\left(\frac{\left(\gamma / \gamma_{1}\right)^{4 / 3}\left\langle 2 \bar{S}_{i j}^{\prime} \bar{S}_{i j}^{\prime}\right\rangle}{1-\beta^{4 / 3}}\right)^{1 / 2}
$$

Hence, based on these definitions, the inertial range behaviour of $v_{t, s 2}^{\prime}$ can be expressed as $R_{s 2} \sim Q_{s 2}-1$.

We now construct a modified VMS model by using the same relation $\mathscr{F}\left(R_{s 2}, Q_{s 2}\right)=0$ as that proposed in (2.28). Elaboration yields

$$
v_{t, s 2}^{\prime *}=\frac{\left(\gamma / \gamma_{2}\right)^{4 / 3}}{1-\beta^{4 / 3}}\left(\sqrt{\left(\frac{C_{s, \infty} \Delta}{\gamma}\right)^{4} \frac{\left(\gamma / \gamma_{1}\right)^{4 / 3}\left\langle 2 \bar{S}_{i j}^{\prime} \bar{S}_{i j}^{\prime}\right\rangle}{1-\beta^{4 / 3}}+v^{2}}-v\right) .
$$

Following a similar procedure, modified models corresponding to (3.1), (3.5) and (3.6) can be constructed. Their associated generalized viscosities are given by

$$
\begin{aligned}
v_{t, s 1}^{\prime *} & =\frac{\left(\gamma / \gamma_{1}\right)^{4 / 3}}{1-\beta^{4 / 3}}\left(\sqrt{\left(\frac{C_{s, \infty} \Delta}{\gamma}\right)^{4} \frac{\left(\gamma / \gamma_{1}\right)^{4 / 3}\left\langle 2 \bar{S}_{i j}^{\prime} \bar{S}_{i j}^{\prime}\right\rangle}{1-\beta^{4 / 3}}+v^{2}}-v\right), \\
v_{t, a 1}^{\prime *} & =\frac{\left(\gamma / \gamma_{1}\right)^{4 / 3}}{1-\beta^{4 / 3}}\left(\sqrt{\left(C_{s, \infty} \Delta / \gamma\right)^{4}\left\langle 2 \bar{S}_{i j} \bar{S}_{i j}\right\rangle+v^{2}}-v\right), \\
v_{t, a 2}^{\prime *} & =\frac{\left(\gamma / \gamma_{2}\right)^{4 / 3}}{1-\beta^{4 / 3}}\left(\sqrt{\left(C_{s, \infty} \Delta / \gamma\right)^{4}\left\langle 2 \bar{S}_{i j} \bar{S}_{i j}\right\rangle+v^{2}}-v\right) .
\end{aligned}
$$

In order to further evaluate the quality of the presented approximations, the dissipation errors $\delta_{\varepsilon}$ will be evaluated for constant-coefficient VMS models (employing 

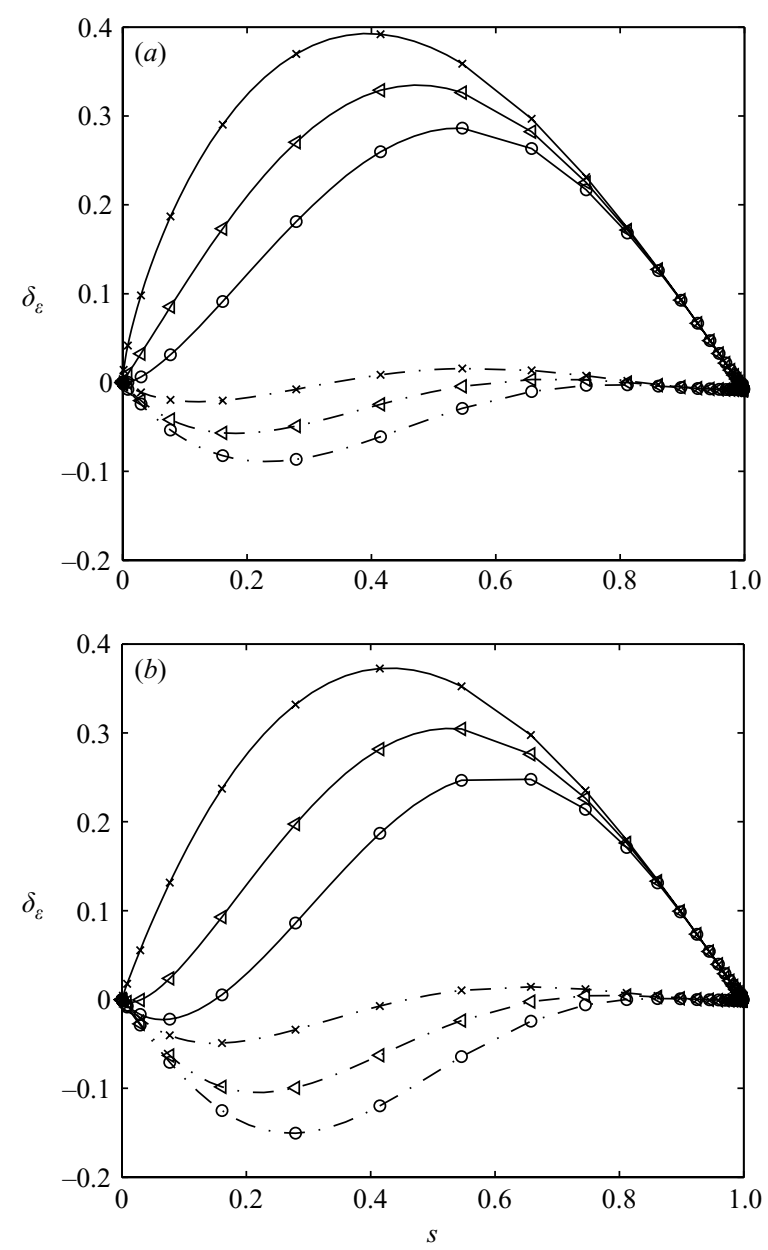

FIGURE 7. Evolution of the errors $\delta_{\varepsilon}^{c t e}$ and $\delta_{\varepsilon}^{*}$ as a function of the subgrid activity $s$ (and $L / \Delta \gg 1)$ for the constant-coefficient $(a)$ small-small and $(b)$ all-small VMS Smagorinsky models and their respective modified versions. -,$\delta_{\varepsilon}^{\text {cte }} ;$ and.,$-- \delta_{\varepsilon}^{*}$. Symbols as in figure 5 .

asymptotic coefficients, cf. e.g. (3.13)), and for the proposed modified VMS models of the current section. To this end, expressions for $\delta_{\varepsilon}$ can be straightforwardly derived, resulting in relations similar to equations (2.31) and (2.32).

In figures $7(a)$ and $7(b)$, the $a$ priori dissipation errors $\delta_{\varepsilon}^{c t e}$ and $\delta_{\varepsilon}^{*}$ are presented as a function of the subgrid activity $s$ (see also the discussion at the end off $\S 2.3$ on how these errors typically show up in actual simulations), and this for the small-small models (figure $7 a$ ) and the all-small models (figure 7b), all employing sharp cutoff filters $\mathscr{H}^{\prime}$ and $\mathscr{G}$. First of all, in figures $7(a)$ and figure $7(b)$, the same behaviour can be observed for both the small-small and all-small formulations, though the main trends are more pronounced for the small-small formulation. Hence, we will direct the discussion of these results mainly to the small-small models.

The dissipation error $\delta_{\varepsilon}^{c t e}$ for the constant-coefficient small-small model in figure $7(b)$ is shown for three different ratios of $\beta$, i.e. $1 / 2,3 / 4$ and 0.95 . We recall that the latter value $(\beta=0.95)$ is added in order to evaluate trends for $\beta \rightarrow 1$, but is not proposed 
as an actual setting for $\beta$ in a practical simulation. All three settings of $\beta$ produce better overall results than the standard Smagorinsky model (cf. figure 4), though the constant-coefficient models remain seriously too dissipative over the full $s$-range. Furthermore, the overall behaviour of the constant coefficient small-small model improves for $\beta \rightarrow 1$. This can be readily explained based on the results previously presented in figure 6 . Because of the non-monotonous behaviour of the exact model coefficients $C_{s 1}$ as a function of $\Delta / \eta$ for higher settings of $\beta$, a constant (asymptotic) coefficient is, for $\Delta / \eta$ around 10, effectively closer to the exact coefficient. Furthermore, the larger deviations around $\Delta / \eta=1$ are not very relevant, since the total model dissipation decreases rapidly in this region. This can partly explain the success of the constant-coefficient VMS models, even if, for example, compared to a dynamic version of the models. In this context, note that in their simulations, Holmen et al. (2004) obtained optimal results (with minimal errors) for the constant-coefficient small-small model at a relatively high setting for $\beta$, i.e. $\beta \approx 0.7$.

Now, the quality of the proposed modifications (3.30) and (3.31) is assessed. For $s$ close to 1 , figure 7 shows that the slope of the error $\delta_{\varepsilon}^{*}$ is zero, in contrast to the slope of $\delta_{\varepsilon}^{\text {cte }}$, which is approximately $-45^{\circ}$. This corresponds to the observations in $\S 2.3$ for the standard Smagorinsky model, and is explained by the better inertial range behaviour of (3.30) and (3.31).

For lower values of $s$, differences related to $\beta$ appear in the errors $\delta_{\varepsilon}^{*}$ of the modified VMS models. This dependence shows an inverse trend when compared to the dependence of $\delta_{\varepsilon}^{\text {cte }}$ on $\beta$, i.e. the quality of the modifications deteriorates for increasing $\beta$. Nevertheless, even for an extreme setting $\beta=0.95$, the errors $\delta_{\varepsilon}^{*}$ remain, in absolute value, lower than $\delta_{\varepsilon}^{\text {cte }}$. At a probably more often selected ratio $\beta=0.5$, we observe $\left|\delta_{\varepsilon}^{*}\right|<5 \%$ compared to $\delta_{\varepsilon}^{\text {cte }}<38 \%$.

\subsection{The effect of the high-pass-filter shape $\mathscr{H}^{\prime}$}

Up until now, only sharp cutoff high-pass filters $\mathscr{H}^{\prime}$ have been used during the evaluation of variational multi-scale models. In this section, the effect of smooth highpass filters will be investigated. We recall that for smooth filters $\mathscr{H}^{\prime}$, the coefficients $C_{s 1}$ and $C_{a 1}$ are not identical anymore to $C_{s 2}$ and $C_{a 2}$ (cf. (3.12), (3.18), (3.17) and (3.19)). However, we verified that differences in behaviour between $C_{s 1}$ and $C_{s 2}$, and between $C_{a 1}$ and $C_{a 2}$ are not very large. In contrast, we will demonstrate that the change of the high-pass filter from a projection filter to a smooth filter results in significant changes in the performance of the models. Moreover, some relevant differences also occur between the small-small and all-small formulations. Therefore, we elected to present results based on (3.2) and (3.6) and their coefficients $C_{s 2}$ and $C_{a 2}$, to demonstrate the main trends. Successively, the dependency of the exact VMS coefficients $C_{s 2}$ and $C_{a 2}$ on the shape of $\mathscr{H}^{\prime}$ is investigated, the quality of the approximations proposed in previous sections is re-established, and the asymptotic $L / \Delta$ behaviour of VMS models based on high-pass projection filters and smooth filters is compared.

Before turning to an extensive analysis of the effect of the filter shape, we recall that for a smooth filter, $\gamma_{1}$ and $\gamma_{2}$ are not equal to one, but correspond to the effective evaluation of equations (3.10) and (3.16). Moreover, for smooth filters, both $\gamma_{1}$ and $\gamma_{2}$ depend on $\beta=\Delta / \Delta^{\prime}$. Hence, where the VMS formulations of the Smagorinsky models are used with smooth high-pass filters $\mathscr{H}^{\prime}$, one should scrupulously figure out their correct values, since they appear in the scaling of $C_{s, \infty}$, not only in the expressions for the exact model coefficients, but also in all the modified models. In figure 8, both $\gamma_{1}$ and $\gamma_{2}$ are displayed as a function of $\beta=\Delta / \Delta^{\prime}$ for 'top-hat' and 


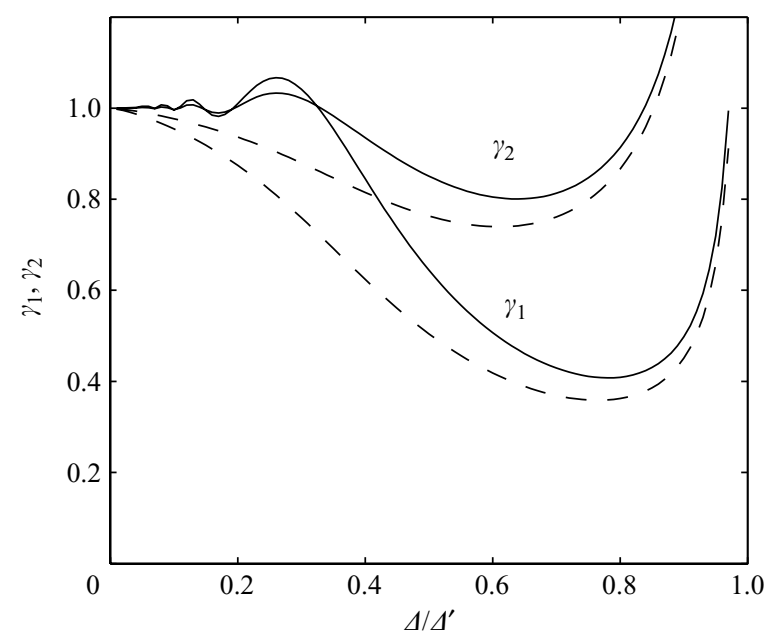

FIGURE 8. The correction coefficients $\gamma_{1}$ and $\gamma_{2}$ as a function of $\beta$, with $\mathscr{G}$ a sharp cutoff filter (with cutoff $\pi / \Delta)$ and the filter $\mathscr{H}^{\prime}$ corresponding to - a 'top-hat' high-pass filter $(H(k)=$ $\left.1-\sin \left[k \Delta^{\prime} / 2\right] /\left[k \Delta^{\prime} / 2\right]\right)$, and ---, a 'Gaussian' high-pass filter $\left(H(k)=1-\exp \left[-k^{2} \Delta^{\prime 2} / 24\right]\right)$.

'Gaussian' high-pass filters (the LES filter $\mathscr{G}$ remains a standard sharp cutoff filter). These filters correspond to high-pass filters, which are constructed by subtracting, respectively, a regular top-hat low-pass filter and Gaussian filter from the identity operator. As can be appreciated from the figure, $\gamma_{1}$ and $\gamma_{2}$ depend strongly on $\beta$ and can be considerably lower than one. Furthermore, for $\beta<0.4$ and a 'top-hat' filter, we can observe oscillatory behaviour for $\gamma_{1}$ and $\gamma_{2}$, which is related to the oscillatory nature of the 'top-hat' filter transfer function. A more extensive analysis of $\gamma_{1}$ and $\gamma_{2}$, specifically addressing filters which are not spherical symmetric in Fourier space (preferably used in many practical implementations), is elaborated in the Appendix.

In figure 9, the evolution of the exact model coefficient $C_{s 2}$ of the small-small model (3.2) is shown both for a sharp cutoff and 'top-hat' high-pass filter. Coefficients of the all-small model show the same trends, but will not be shown. In order to compare the coefficients $C_{s 2}$, they are appropriately normalized by multiplying them with $\left(1-\beta^{4 / 3}\right)^{3 / 4} \gamma_{1}^{1 / 3} \gamma_{2}^{2 / 3}$ (cf. equation (3.17)). Three different ratios $\Delta / \Delta^{\prime}$ are shown, corresponding to $0.5,0.75$ and 0.95 . From figure 9 , we can appreciate that the normalized model coefficients for 'top-hat' high-pass filtered VMS models, are insensitive to the LES-to-high-pass filter ratio $\beta$. For $\beta=0.75$ and 0.95 , values are visually the same, while for $\beta=0.5$, differences are not very large. This is in clear contrast to the behaviour of the normalized coefficients related to sharp cutoff highpass filtered VMS models, which are also shown in the figure.

The large disparity in behaviour of the model coefficients for these two high-pass filters is related to the 'top-hat' high-pass filter being a smooth filter, which does not 'cut' away scales, but rather smoothly dampens certain scales. It is well known that a 'top-hat' high-pass filter $\mathscr{H}_{t h}^{\prime}$ is a second-order filter. Consequently, we can trivially show that

$$
\int_{0}^{2 \pi / \ell} H_{t h}^{\prime}(k) \phi(k) \mathrm{d} k \sim \Delta^{\prime 2}, \quad \int_{0}^{2 \pi / \ell}\left(H_{t h}^{\prime}(k)\right)^{2} \phi(k) \mathrm{d} k \sim \Delta^{\prime 2},
$$

with $\ell \gg \Delta^{\prime}$, an arbitrary constant length, and $\phi$ an arbitrary function. Hence, even if the inertial subrange and the main point of dissipation are situated at scales 


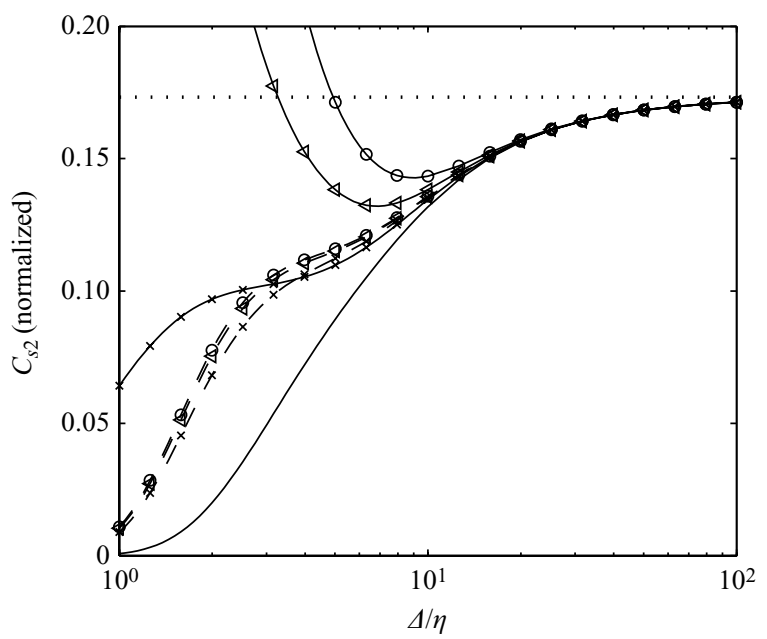

FIGURE 9. Evolution of $C_{s 2}$ (normalized) as function of the ratio $\Delta / \eta$ for different values of $\Delta / \Delta^{\prime}$, and two different high-pass filters $\mathscr{H}^{\prime}(L / \Delta \gg 1)$. $-C_{s}\left(=C_{s 2}\right.$ for $\left.\Delta / \Delta^{\prime}=0\right)$. Curves with symbols: -,$C_{s 2}$ evaluated with a cutoff high-pass filter $\mathscr{H}^{\prime}$ for different $\Delta / \Delta^{\prime}$; - - $C_{s 2}$ evaluated with a 'top-hat' high-pass filter $\mathscr{H}^{\prime}$ for different $\Delta / \Delta^{\prime}$. Symbols as in figure 5.

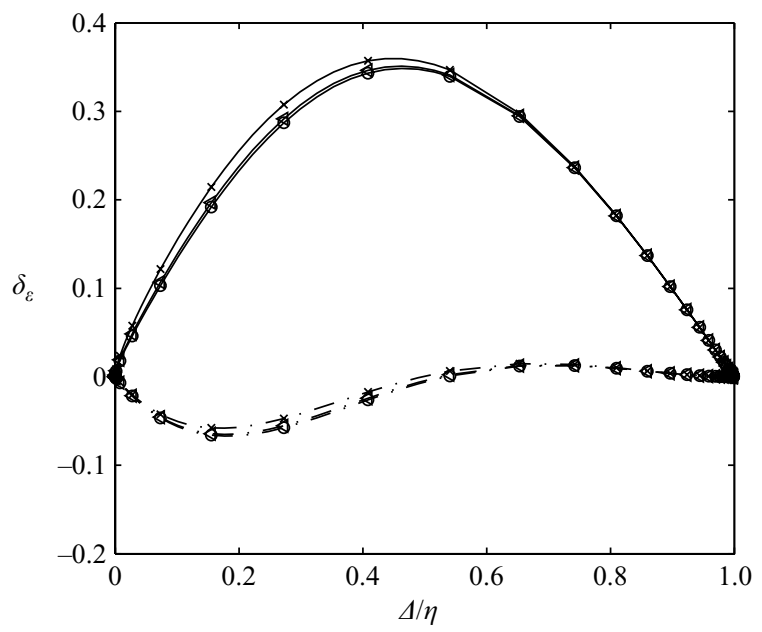

Figure 10. Evolution of the errors $\delta_{\varepsilon}^{c t e}$ and $\delta_{\varepsilon}^{*}$ as a function of the subgrid activity $s$ (and $L / \Delta \gg 1)$ for the constant-coefficient small-small VMS Smagorinsky model (3.2) and its modified version (3.29), all using a 'top-hat' high-pass filter $\mathscr{H}^{\prime} .-, \delta_{\varepsilon}^{\text {cte }} ;-\cdot-, \delta_{\varepsilon}^{*}$. Symbols as in figure 5.

considerably larger than $\Delta^{\prime}$, their effect on the integral in (3.8), or (3.15), scales with $\Delta^{\prime 2}$. In contrast, the far end of Kolmogorov's equilibrium range (at scales around and smaller than the Kolmogorov scale $\eta$ ) decays exponentially. Consequently, when $\Delta / \Delta^{\prime}$ increases, the contribution of the inertial subrange and the main point of dissipation will remain relatively important in the total integral.

The quality of the proposed modifications to the VMS models for a 'top-hat' high-pass filter is addressed in figure 10, based on an evaluation of the relative dissipation errors $\delta_{\varepsilon}^{c t e}$ and $\delta_{\varepsilon}^{*}$. Only results for the small-small models are shown, as trends for the all-small model are similar. Results in figure 10 affirm the quality of 


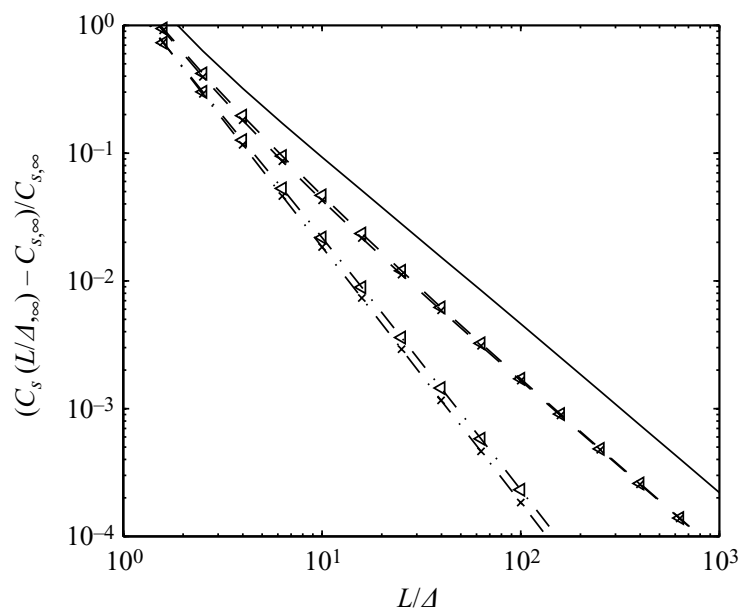

FIGURE 11. Deviation of $C_{s}, C_{s 2}$ (normalized) and $C_{a 2}$ (normalized) from the asymptotic value $C_{s, \infty}$ as a function of $L / \Delta$ for $\operatorname{Re}_{L}=\infty$ and different high-pass filters $\mathscr{H}^{\prime}$. The deviations are normalized using $C_{s, \infty}$. -,$C_{s} ;-.-, C_{s 2} ;---, C_{a 2}$. Symbols further correspond to $\times$, cutoff high-pass filter with $\Delta / \Delta^{\prime}=1 / 2 ; \triangleleft$, 'top-hat' high-pass filter with $\Delta / \Delta^{\prime}=1 / 2$.

the proposed approximations to the behaviour of the exact model (i.e. using exact coefficients). Moreover, in contrast to trends observed for a sharp cutoff high-pass filter (cf. figure 7), the quality of the models is quite insensitive to $\Delta / \Delta^{\prime}$ ' for 'top-hat' high-pass filtered VMS. Hence, the use of 'top-hat' high-pass filters provides more robust formulations of the VMS Smagorinsky models. It is inspiring to observe that Sagaut \& Levasseur (2005), based on an extensive sensitivity analysis, found that (constant-coefficient) VMS models employing a smooth high-pass filter instead of projection filters, effectively yield improved results in simulations.

Finally, the asymptotic $L / \Delta$ behaviour of the VMS models employing a 'top-hat' high-pass filter, is investigated. In figure 11, the results are presented for the smallsmall (3.17) and all-small (3.19) VMS formulations employing a cutoff and a 'top-hat' high-pass filter, with $\Delta / \Delta^{\prime}=0.5$. As can be seen from the figure, the change of high-pass filters does not significantly influence the results. With respect to the slopes associated to the asymptotic $L / \Delta$ behaviour, an analysis similar to that presented in $\S 3.3$ (cf. equations (3.22)-(3.24)) can be elaborated. If we take $q$ as the order of the high-pass filter, we can verify that

$$
\begin{aligned}
\delta C_{a 2} & \sim(L / \Delta)^{-\min (4 / 3,4 / 3+q, p)} \stackrel{(q>0)}{\sim}(L / \Delta)^{-\min (4 / 3, p)}, \\
\delta C_{s 2} & \sim(L / \Delta)^{-\min (4 / 3+q, p)},
\end{aligned}
$$

where the second proportionality in (3.34) results from the order $q$ being assumed to be larger than 0 , such that $\min (4 / 3,4 / 3+q, p)=\min (4 / 3, p)$. Hence, the use of a second-order top-hat filter $(q=2)$ does not change anything for the $L / \Delta$ asymptotic behaviour of the all-small model. For the small-small model, a smooth filter does not change anything, provided $q>p-4 / 3$. For $p=2$, associated with Pope's spectrum, and $q=2$, this condition is satisfied.

\section{Conclusions}

In the current paper, an exhaustive analysis has been presented on the required behaviour of model coefficients, belonging to the standard and variational multi-scale 
formulations of the Smagorinsky model, as a function of the subgrid activity, the ratio $\Delta / \eta$, and as a function of $L / \Delta$. Moreover, modifications to the Smagorinsky model and its VMS formulations are proposed, i.e. specifically recall equations (2.29), and (3.29)-(3.32). In a priori analyses employing well-established spectrum descriptions, these modifications showed considerable improvement, compared to the classical constant-coefficient versions.

The applied methodology in the present paper, is an extension of Lilly's (1967) method for the determination of his 'universal' Smagorinsky constant. Instead of using a simplified (high-Reynolds-number) $-5 / 3$ shape of the spectrum, as Lilly employed in his analysis, here, general Reynolds-dependent spectra are premised, which include an energy-containing large-scale range, an inertial subrange, and a dissipation range. Central to the formulation of a surveyable and elegant methodology are the definitions of three normalized functions $\Phi, \Psi_{1}$ and $\Psi_{2}$, which incorporate the effects of the shape of the spectrum and the different filters, and can be easily used for the construction and evaluation of relations describing the behaviour of the different coefficients. Some practical evaluations and verifications of the proposed relations are based on Pope's (2000) spectra. However, all theoretical results are presented in a generic way, which is independent of the definition or use of a particular choice of spectrum.

The evaluation of the standard Smagorinsky coefficient as a function of $\Delta / \eta$ (the ratio between the filter width and the Kolmogorov scale) reveals that the coefficient decreases rapidly when $\Delta / \eta \rightarrow 1$. These results are in line with the observations by Voke (1996). Moreover, by evaluating the coefficient as a function of the subgrid activity, which is a nonlinear transformation of $\Delta / \eta$, we can appreciate that the Smagorinsky coefficient - even in the vicinity of $s=1-$ is not constant.

Therefore, a framework has been elaborated which allows the development of easy-to-use generic modifications to the original model, such that the effective model coefficients of the modified model follow the behaviour of the 'exact' coefficients better. For the Smagorinsky model, we propose to express the eddy viscosity $v_{t}$ in large-eddy simulations $v_{t}=\left(v_{\text {Lilly }}^{2}+v^{2}\right)^{1 / 2}-v$ (cf. 2.29), with $v_{\text {Lilly }}$ the turbulent viscosity obtained by employing Lilly's model constant and $v$ the laminar viscosity. Analysis of the constant-coefficient model and the proposed modification reveals the quality of the improvements. First of all, the constant-coefficient model is clearly too dissipative over the full subgrid-activity range $(0<s<1)$, with a priori errors on the total dissipation up to $50 \%$. In contrast, the proposed modification was shown to be very accurate for all settings of $s$, with errors below $5 \%$.

Next to the standard Smagorinsky model, similar analyses have been performed for the small-small and all-small variational multi-scale formulations of the Smagorinsky model (Hughes et al. 2000; Holmen et al. 2004). Overall, trends observed for the standard Smagorinsky model also show for the VMS models, though an additional dependence of the coefficients on $\Delta / \Delta^{\prime}$ (expressing the ratio of the LES-filter width $\Delta$ to the VMS-high-pass-filter width $\Delta^{\prime}$ ) is observed. Modified models have been presented in equations (3.30) and (3.29) for the small-small model, and in equations (3.31) and (3.32) for the all-small model. Furthermore, the quality of the improvements has been rigorously assessed.

The relevance of the various proposed modifications is, in our opinion, evident. As established based on an a priori evaluation, these modifications contain a good 'built-in' $\Delta / \eta$ scaling, and are, at the same time, easy to use in actual implementations, i.e. they can be operated using asymptotically determined constant coefficients. Consequently, the robustness of these models and their application range can be considerably larger than that of their standard constant-coefficient counterparts. 
Obviously, these prospects need evaluation in various practical test cases, and hence contain material for further work.

Next to the extensive analysis of the $\Delta / \eta$ dependence of the model coefficients, also the influence of $L / \Delta$ on the model has been investigated, with $L$ the length scale related to the energy-containing large scales. In $\S 1$, we hypothesized that a comparison of the asymptotic $L / \Delta$ behaviour of subgrid-scale models might elucidate their relative quality. Consequently, this asymptotic behaviour was investigated for the standard and both VMS Smagorinsky models. First of all, if an arbitrary deviation of the model coefficient from its asymptotic value is used as a reference, we can observe that the Smagorinsky model always requires the highest ratio $L / \Delta$ to attain this value, followed by the all-small model, while the small-small model requires the lowest $L / \Delta$ (e.g. for a deviation of $1 \%$, ratios are, respectively, $L / \Delta=55,25$ and 10). Furthermore, we evaluated the slope of the asymptotic convergence at high ratios $L / \Delta$ for the three models. It turned out that the slope of convergence of the Smagorinsky model and the all-small Smagorinsky model are the same, amounting to $-\max \{4 / 3, p\}$, with $p$ the decay rate of the large-scale influence in the inertial subrange. For the small-small model, we found this slope always to be $-p$, corresponding to the steepest physically attainable slope.

Finally, for the VMS formulations, the change from high-pass projection filters, as originally proposed in the variational multi-scale framework by Hughes et al. (2000), towards smooth filters, as tested by Vreman (2003), is investigated. It has been surprising to find that smooth high-pass filters improve the 'robustness' of the VMS models. Furthermore, provided some constraints are set on the order of the high-pass filters, the $L / \Delta$ asymptotic behaviour does not change significantly.

\section{Appendix. Weighting coefficients for filters which are not spherical-symmetric in Fourier space}

All derivations in the main text of the present paper implicitly assume that the filters $\mathscr{G}$ and $\mathscr{H}^{\prime}$ are spherical symmetric in Fourier-space, such that their one-dimensional transfer function can be used directly for the filtering of the shell-integrated energy spectrum. This Appendix briefly addresses the issues which arise, when filters are used which are not spherical symmetric in Fourier space, since these types of filters often occur in practical LES implementations.

First, where the LES filter $\mathscr{G}$ is not spherical symmetric in Fourier space, the definition of the function $\Phi(2.13)$ must be replaced by a three-dimensional equivalent, i.e.

$$
\Phi\left(\frac{L}{\Delta}, R e_{L}\right)=\frac{\frac{1}{4 \pi} \int_{0}^{\infty} x^{1 / 3} f_{L}(x) f_{\eta}\left(x R e_{L}^{-3 / 4}\right)\left[\int_{0}^{2 \pi} \int_{0}^{\pi} G(x / L)^{2} \sin \theta \mathrm{d} \theta \mathrm{d} \phi\right] \mathrm{d} x}{\frac{1}{4 \pi} \int_{0}^{\infty} \int_{0}^{2 \pi} \int_{0}^{\pi} x^{1 / 3} G(x / L)^{2} \sin \theta \mathrm{d} \theta \mathrm{d} \phi \mathrm{d} x},
$$

with $\boldsymbol{x}=\boldsymbol{k} L=\left[k_{1} L, k_{2} L, k_{3} L\right]$ the normalized wave vector in Fourier space. Using a spherical coordinate system, we can ensure that $x=[x \sin \theta \cos \phi, x \sin \theta \sin \phi, x \cos \theta]$, with $x$ the magnitude of the vector $x, \phi$ the azimuth, and $\theta$ the zenith.

Expressions equivalent to (A 1) can be readily derived for $\Psi_{1}$ and $\Psi_{2}$. Of more direct practical importance though, is the formulation of the weighting coefficients $\gamma, \gamma_{1}$ and $\gamma_{2}$, as they appear as scaling factors in the modified models (2.29) and (3.29)-(3.32). 


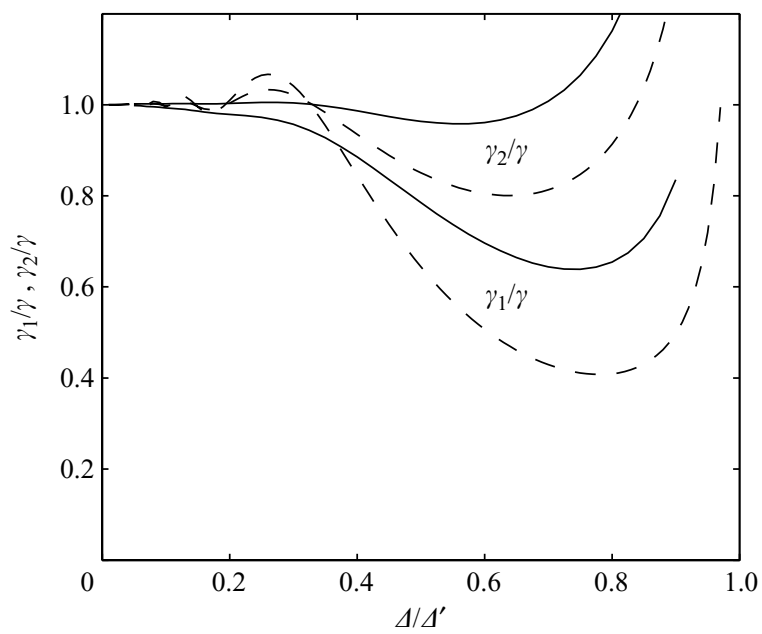

FIGURE 12. The correction coefficients $\gamma_{1} / \gamma$ and $\gamma_{2} / \gamma$ as a function of $\beta$, both for filters which are spherical-symmetric and non-spherical-symmetric in Fourier space. - - -, $\mathscr{H}^{\prime}$ a 'top-hat' high-pass filter and $\mathscr{G}$ a cutoff filter (with cutoff $\pi / \Delta$ ), both spherical symmetric; -, $\mathscr{H}^{\prime}$ corresponding to (A 5) and $\mathscr{G}$ a cubical cutoff filter (with cutoff $\pi / \Delta$ ).

One easily obtains

$$
\begin{gathered}
\gamma=\left(\frac{1}{3 \pi} \frac{\int_{0}^{\infty} \int_{0}^{2 \pi} \int_{0}^{\pi} k^{1 / 3}(G(\boldsymbol{k}))^{2} \sin \theta \mathrm{d} \theta \mathrm{d} \phi \mathrm{d} k}{(\pi / \Delta)^{4 / 3}}\right)^{3 / 4}, \\
\gamma_{1}=\left(\frac{1}{3 \pi} \frac{\int_{0}^{\infty} \int_{0}^{2 \pi} \int_{0}^{\pi} k^{1 / 3}\left(H^{\prime}(\boldsymbol{k})\right)^{2}(G(\boldsymbol{k}))^{2} \sin \theta \mathrm{d} \theta \mathrm{d} \phi \mathrm{d} k}{(\pi / \Delta)^{4 / 3}\left(1-\beta^{4 / 3}\right)}\right)^{3 / 4} .
\end{gathered}
$$

and

$$
\gamma_{2}=\left(\frac{1}{3 \pi} \frac{\int_{0}^{\infty} \int_{0}^{2 \pi} \int_{0}^{\pi} k^{1 / 3} H^{\prime}(\boldsymbol{k})(G(\boldsymbol{k}))^{2} \sin \theta \mathrm{d} \theta \mathrm{d} \phi \mathrm{d} k}{(\pi / \Delta)^{4 / 3}\left(1-\beta^{4 / 3}\right)}\right)^{3 / 4},
$$

We will briefly discuss some results, obtained from the numerical integration of these equations using a few selected filters.

The integration of (A 2) for a cubical sharp cutoff filter with cutoff $\pi / \Delta$ leads to $\gamma \approx 1.22$. This is significantly higher than $\gamma=1$ for a spherical cutoff filter. This difference reflects the fact, that a cubical cutoff filter, for the same value of $\Delta$, retains overall more scales than the spherical cutoff filter. In fact, $\gamma$ provides a link between the formal - often filter dependent - definition of the filter width $\Delta$, and the 'effective' filter width of its accompanying filter. In fact, one might have recognized in all modified models, that $\gamma$ appears as a scaling of $\Delta$.

Likewise, equations (A 3) and (A 4) can be integrated numerically. In figure 12, we present, as a function of $\Delta / \Delta^{\prime}$, results for a cubical cutoff filter $\mathscr{G}$ combined with a 
top-hat filter defined as

$$
H_{t h}^{\prime}(\boldsymbol{k})=1-\prod_{i=1}^{3} \frac{\sin \left(k_{i} \Delta^{\prime} / 2\right)}{k_{i} \Delta^{\prime} / 2} .
$$

Since both $\gamma_{1}$ and $\gamma_{2}$ are scaled by $\gamma$ in all modified equations, $\gamma_{1} / \gamma$ and $\gamma_{2} / \gamma$ are represented in figure 12. This scaling ensures (cf. their respective definitions) that $\gamma_{1} / \gamma$ and $\gamma_{2} / \gamma$ are 1 for $\beta=0$. Furthermore, as a point of reference, the weighting factors associated with the spherical-symmetric equivalents of $\mathscr{G}$ and $\mathscr{H}^{\prime}$ are also displayed. It is clear that the oscillatory behaviour of $\gamma_{1}$ and $\gamma_{2}$ for the spherical-symmetric 'top-hat' filter does not occur for (A 5), since the oscillations in (A 5) are not aligned with wavenumber spheres in Fourier space.

\section{REFERENCES}

Bou-Zeid, E., Meneveau, C. \& Parlange, M. 2005 A scale-dependent Lagrangian dynamic model for large eddy simulation of complex turbulent flows. Phys. Fluids 17 (2), 025105.

Canuto, V. M. \& Cheng, Y. 1997 Determination of the Smagorinsky-Lilly constant $C_{s}$. Phys. Fluids $9(5), 1368-1378$.

Carati, D., Winckelmans, G. S. \& Jeanmart, H. 2001 On the modelling of the subgrid-scale and filtered-scale stress tensors in large-eddy simulation. J. Fluid Mech. 441, 119-138.

DEARdofF, J. W. 1970 A numerical study of three-dimensional turbulent channel flow at large Reynolds numbers. J. Fluid Mech. 41, 453-480.

Domaradzki, J. A. \& Rogallo, R. S. 1990 Local energy transfer and nonlocal interactions in homogeneous, isotropic turbulence. Phys. Fluids A 2 (3), 413-426.

Fureby, C. \& TABOR, G. 1997 Mathematical and physical constraints on large-eddy simulations. Theor. Comput. Fluid Dyn. 9 (2), 85-102.

Germano, M., Piomelli, U., Moin, P. \& Савот, W. H. 1991 A dynamic subgrid-scale eddy viscosity model. Phys. Fluids A 3 (7).

GeurTs, B. J. 2003 Elements of Direct and Large-Edddy Simulation. R.T. Edwards, Flourtown.

GeurTs, B. J. \& FröHLICH, J. 2002 A framework for predicting accuracy limitations in large eddy simulations. Phys. Fluids 14 (6), L41-L44.

Ghosal, S. 1999 Mathematical and physical constraints on large-eddy simulation of turbulence. AIAA J. 37, 425-433.

HinZe, J. O. 1959 Turbulence: An Introduction to Its Mechanism and Theory. McGraw-Hill.

Holmen, J., Hughes, T. J. R., Oberai, A. A. \& Wells, G. N. 2004 Sensitivity of the scale partition for variational multiscale large-eddy simulation of channel flow. Phys. Fluids 16 (3), 824-827.

Hughes, T. J. R., Mazzei, L. \& Jansen, K. E. 2000 Large eddy simulation and the variational multiscale method. Comput. Visualiz. Sci. 3(1.2), 47-59.

Hughes, T. J. R., Mazzei, L. \& Oberai, A. A. 2001a The multiscale formulation of large eddy simulation: decay of homogeneous isotropic turbulence. Phys. Fluids 13 (2), 505-512.

Hughes, T. J. R., Oberai, A. A. \& Mazzei, L. $2001 b$ Large eddy simulation of turbulent channel flows by the variational multiscale method. Phys. Fluids 13 (6), 1784-1799.

Jeanmeart, H. \& Winckelmans, G. S. 2002 Comparison of recent dynamic subgrid-scale models in turbulent channel flow. In Studying Turbulence Using Numerical Simulation Databases IX, pp. 105-116. CTR, Stanford.

JimÉNEZ, J. 2003 Computing high-Reynolds-number turbulence: will simulations ever replace experiments? J. Turbulence 4, 022.

JimÉNEZ, J. \& Moser, R. D. 2000 Large-eddy simulations: where are we and what can we expect? AIAA J. 38, 605-612.

Kraichnan, R. H. 1976 Eddy viscosity in two and three dimensions. J. Atmos. Sci. 33, 1512-1536.

Lesieur, M. \& MÉtais, O. 1996 New trends in large-eddy simulations of turbulence. Annu. Rev. Fluid Mech. 28, 45-82.

Leslie, D. C. \& Quarini, G. L. 1979 The application of turbulence theory to the formulation of subgrid modelling procedures. J. Fluid Mech. 91, 65-91. 
LiLLY, D. K. 1966 On the application of the eddy-viscosity concept in the inertial sub-range of turbulence. Tech. Rep. 123. NCAR.

LiLlY, D. K. 1967 The representation of small-scale turbulence in numerical simulation experiments. In Proc. IBM Scientific Computing Symposium on Environmental Sciences. IBM Data Processing Division, White Plains, New York.

McMillan, O. J. \& Ferziger, J. H. 1979 Direct testing of subgrid-scale models. AIAA J. 17, $1340-1346$.

Magnient, J.-C., Sagaut, P. \& Deville, M. 2001 A study of built-in filter for some eddy viscosity models in large-eddy simulation. Phys. Fluids 13 (5), 1440-1449.

Meneveau, C. 1994 Statistics of turbulence subgrid-scale stresses: necessary conditions and experimental tests. Phys. Fluids 6 (2), 815-833.

Meneveau, C. \& Lund, T. S. 1997 The dynamic Smagorinsky model and scale-dependent coefficients in the viscous range of turbulence. Phys. Fluids 9 (12), 3932-3934.

Meyers, J. \& Baelmans, M. 2004 Determination of subfilter energy in large-eddy simulations. J. Turbulence 5 (026).

Meyers, J., Geurts, B. J. \& Baelmans, M. 2003 Database-analysis of errors in large-eddy simulation. Phys. Fluids 15 (9), 2740-2755.

MoIn, P. \& KIM, J. 1997 Tackling turbulence with supercomputers. Sci. Am. 276, 62-68.

MuschinsKi, A. 1996 A similarity theory of locally homogeneous and isotropic turbulence generated by a Smagorinsky-type LES. J. Fluid Mech. 325, 239-260.

Pope, S. B. 2000 Turbulent Flows. Cambridge University Press.

Pope, S. B. 2004 Ten questions concerning the large-eddy simulation of turbulent flows. New J. Phys. 6, 35.

Porté-Agel, F., Meneveau, C. \& Parlange, M. B. 2000 A scale-dependent dynamic model for large-eddy simulation: application to a neutral atmosoheric boundary layer. J. Fluid Mech. 415, 261-284.

SaGaut, P. 2002 Large Eddy Simulations for Incompressible Flows, 2nd edn. Springer.

SAGAut, P. \& LeVASSEUR, V. 2005 Sensitivity of spectral variational multiscale methods for large-eddy simulation of isotropic turbulence. Phys. Fluids 17 (3), 035113.

SMAGORINSKY, J. 1963 General circulation experiments with the primitive equations: I. The basic experiment. Mon. Weather Rev. 91, 99-165.

Stolz, S., Schlatter, P. \& Kleiser, L. 2005 High-pass filtered eddy-viscosity models for large-eddy simulations of transitional and turbulent flow. Phys. Fluids 17, 065103.

Suk Kang, H., Chester, S. \& Meneveau, C. 2003 Decaying turbulence in an active-grid-generated flow and comparisons with large-eddy simulation. J. Fluid Mech. 480, 129-160.

VoKe, P. R. 1996 Subgrid-scale modelling at low mesh Reynolds number. Theoret. Comput. Fluid Dyn. 8, 131-143.

VReman, A. W. 2003 The filtering analog of the variational multiscale method in large-eddy simulation. Phys. Fluids 15 (8), L61-L64.

VReman, A. W. 2004 The adjoint filter operator in large-eddy simulation of turbulent flow. Phys. Fluids 16 (6), 2012-2022.

Vreman, B., Geurts, B. J. \& Kuerten, H. 1994 Realizability conditions for the turbulent stress tensor in large-eddy simulation. J. Fluid Mech. 278, 351-362. 